\title{
FLUCTUACIONES CLIMATICAS Y VARIABILIDAD TEMPORAL DEL CLIMA EN EL NORTE ARGENTINO - 1931/2005
}

Prof. María Emilia Pérez

\author{
Prof. Titular Cátedra: Seminario de Fisiografía - Prof. Adjunta Cátedra: Climatología \\ Dpto. Geografía - Facultad de Humanidades - UNNE
}

Conferencia dictada en el XI ENCUENTRO DE PROFESORES EN GEOGRAFIA DEL NORDESTE. Departamento de Geografía, Facultad de Humanidades, UNNE. Resistencia, 23 de septiembre de 2006.

La Climatología puede definirse como "la ciencia que se ocupa del estudio de la distribución de los climas sobre la superficie terrestre y de sus relaciones con los restantes componentes del medio geográfico", de ahí que actualmente, el clima es considerado como un complejo sistema de interrelaciones entre variables y procesos, que tienen lugar en ámbitos tan diferentes como la atmósfera, la hidrosfera (océanos, mares y cuerpos de agua), la litosfera (superficie terrestre), la criosfera (capas de hielo y nieve) y la biosfera (los ecosistemas).

Sus elementos son los componentes que lo definen (temperatura, precipitación, presión, etc), y las variables a través de las cuales se manifiesta su influencia sobre los demás elementos del medio natural. Como variable climática, los elementos nos permiten definir y caracterizar el clima de una región y determinar los mecanismos que lo condicionan.

La importancia del clima para la sociedad radica en que el valor de su estado medio o condición, su variación en el tiempo (en escala diaria, estacional, anual, decenal y secular), su carácter y extensión geográfica, la frecuencia y persistencia de sus valores extremos, determinan la disponibilidad de recursos de los cuales depende la humanidad.

Ahora bien, la noción de clima expresa el conjunto de tendencias resultantes de las condiciones habituales y medias durante un largo período, que como mínimo se suele establecer en treinta años, y que se consideran relativamente estable o en equilibrio global dentro del sistema climático, lo que a su vez, se traduce en la estabilidad que presentan los climas de la Tierra, por lo menos a escala humana e histórica.

Sin embargo, este hecho no supone ni significa que el clima sea invariable en el tiempo y en el espacio, porque los valores medios sólo tienen un valor relativo, ya que las principales características que definen a los elementos climáticos es su variabilidad natural, que se manifiesta a través de:

- la variabilidad espacial, que explica las diferencias regionales de los climas sobre la superficie terrestre. Esta variabilidad está determinada por la influencia de los elementos y factores astronómicos y geográficos (la latitud, altitud, efecto marítimo o continental, corrientes marinas). Los primeros condicionan los rasgos climáticos dominantes en grandes áreas geográficas, los segundos los modifican.

- la variabilidad temporal, que puede ser de orden diario, mensual, estacional, anual, o en intervalos temporales más largos (décadas, centurias, milenios). La variabilidad temporal se relaciona con los factores cósmicos: movimientos de rotación y traslación de la Tierra, su posición con respecto del sol, desplazamiento estacional de los grandes sistemas de presión y flujos de aires, entre otros.

El acceso a los registros climáticos se ha ampliado durante las últimas décadas, lo cual incrementó la oportunidad de encontrar tendencias y variaciones que parecen ser más significativas a medida que los datos aumentan. De allí que las investigaciones más modernas presenten evidencias que sugieren la noción de que un clima estático no es largamente constante, y consecuentemente, se ha comenzado a comprender que 30 años de datos, el período que ha sido usado para calcular las "normales" de temperatura y precipitación, tiene una longitud inadecuada para definir el clima y abarcar todas las posibles fluctuaciones que sus elementos presentan en esa escala temporal, es decir, no nos provee de información suficiente como para cubrir la amplia variabilidad que posee el clima de la Tierra en escalas temporales de décadas a siglos.

De hecho, las condiciones del sistema climático oscilan alrededor de un valor medio que tiende a mantener una trayectoria permanente a través del tiempo, aunque sometida a continuos ajustes internos o controles reguladores para conseguir un estado estable, originándose de esta manera, una combinación de estabilidad y variaciones en el tiempo, que ha llevado a manifestar a Cuadrat y Pita (1997), que "el sistema climático terrestre es un sistema dinámico en equilibrio transitorio", y a 
Fernández García (1996), que "lo que caracteriza al clima y a sus elementos es un ordenado desorden".

Un aspecto del clima que actualmente resulta de sumo interés, tanto para los especialistas en la materia como para los que no lo son, son las diversas especulaciones existentes acerca de su evolución, en relación con la posibilidad de un cambio climático, que para algunos ya está en marcha por efecto antrópico. Pero si bien es una parte muy atrayente e importante de la investigación meteorológica, también es una de las más inciertas, expresan Barry y Chorley (1985)

El interés actual por el clima se fundamente en que estamos ante la primera crisis climática desde que la Climatología se ha convertido en disciplina científica y por la convicción del papel desempeñado por el hombre sobre esta crisis. Hasta la década 1840/1850 los estudiosos no se habían dado cuenta que el clima había sufrido cambios a lo largo del tiempo, pero a partir de esos años, los avances, fundamentalmente en la física, en las ciencias naturales y en la geología (a través del estudio y datación de fósiles), permitieron obtener pruebas irrefutables de la existencia de climas muy diferentes en distintas épocas geológicas, tales como las condiciones glaciales presentes en la historia de la Tierra.

No obstante, los únicos datos disponibles dignos de confianza, son los obtenidos durante los últimos cien años, a través de la observación y medición de los parámetros meteorológicos, por lo que sólo es posible investigar de manera adecuada las fluctuaciones climáticas recientes. Y porqué especificamos o nos referimos a la expresión fluctuaciones climáticas recientes, porque consideramos que, desde el punto de vista de la Climatología, las diferencias observadas y detectadas en los parámetros meteorológicos corresponden, según su magnitud y duración, a fluctuaciones, variaciones u oscilaciones climáticas y no a cambios climáticos en sentido estricto, como es tan común escuchar y leer en las noticias de actualidad. Por lo tanto, en primer lugar, vamos a realizar a continuación, algunas aclaraciones desde el punto de vista conceptual.

La única fuente de información directa con que cuenta la Climatología es la observación y medición de los diferentes parámetros meteorológicos. El conjunto de observaciones realizadas en las distintas estaciones meteorológicas forman las series climáticas, que son la información básica necesaria para el estudio del clima.

El clima de un lugar es, según Cuadrat y Pita (1997) "la generalización estadística de su comportamiento atmosférico, considerado éste como estable o estacionario, aunque enormemente variable en el tiempo". Este sistema con su funcionamiento, genera como resultado el mosaico climático mundial, que es estable, lo que a su vez se traduce en el hecho de que el sistema es un sistema en equilibrio, pero a su vez es variable "dado que el equilibrio del sistema no es un equilibrio estático sino dinámico".

Ahora bien, esto no ha sido siempre así; existen numerosas evidencias que demuestran que en épocas pasadas, los climas de la Tierra presentaban una configuración espacial diferente de la actual (pinturas rupestres expresivas de paisajes de sabanas en el actual desierto del Sahara, reliquias de vegetación criófila en zonas hoy cálidas, etc.). Desde hace tiempo se conoce que en un pasado remoto se produjeron lentos pero muy importantes cambios en el clima y, a grandes rasgos, se puede diferenciar entre:

a. cambios climáticos producidos en épocas geológicas: son los que ocurrieron durante el precámbrico, el paleozoico superior y los períodos glaciales de fines del mesozoico y principios del cuaternario,

b. cambios u oscilaciones que se efectuaron en épocas históricas: son los que comprenden los últimos 10.000 años, "breve período geológico hasta nuestros días", en el cual los índices paleoclimáticos indican que la Tierra conoció condiciones climáticas cambiantes, pero dentro de límites mucho más estrechos que los anteriores, caracterizados por ritmos de expansión y recesión de los hielos polares y de los glaciares de montaña, a intervalos de 2.000 a 3.000 años, conocidos como "ciclos neoglaciales", y

c. variaciones y fluctuaciones climáticas recientes: son las que comprenden los años posteriores a 1850 hasta la actualidad.

Debido a que las escalas temporales de variación que presenta el sistema climático son muchas: anuales, estacionales, decenales, seculares, milenarias, etc. se dificulta, no sólo la identificación, sino la propia conceptualización de la expresión "cambio climático", lo cual se refleja en la diversa nomenclatura existente al respecto. Incluso la clasificación propuesta por la Organización 
Meteorológica Mundial, que utiliza nueve conceptos diferentes para definir este hecho, atribuye a la expresión "cambio climático" un carácter general, válido para englobar todas las formas de "inconstancias climáticas" cualquiera sea su naturaleza estadística y su causa, configurando una nomenclatura tan abundante y detallada como confusa, tal como manifiestan Cuadrat y Pita (1997):

1. Cambio Climático: término general que engloba todas las formas de inconstancia climática, cualquiera que sea su naturaleza estadística (o su causa física).

2. Discontinuidad climática: cambio climático que consiste en una modificación más bien brusca y permanente de un valor "medio" a otro, acaecida durante el período de observación.

3. Fluctuación climática: inconstancia climática que consiste en cualquier forma de modificación sistemática, sea regular o irregular, a excepción de la "tendencia" o de la "discontinuidad". Está caracterizada por al menos dos máximos (o mínimos) y un mínimo (o máximo), incluidos los situados al principio y al final de la serie.

4. Oscilación climática: "fluctuación" en la cual la variable tiende a cambiar gradual y regularmente entre máximos y mínimos sucesivos (por oposición a vacilación). ${ }^{4}$

5. Periodicidad climática: "ritmo" en el cual el intervalo de tiempo entre máximos y mínimos sucesivos es constante o casi constante durante todo el período de observaciones.

6. Ritmo climático: "oscilación" o "vacilación" en la cual los máximos y los mínimos se presentan a intervalos de tiempo aproximadamente iguales.

7. Tendencia climática: "cambio climático" caracterizado por una disminución o un aumento regulares y monótonos de los valores medios durante el período de observación. El término no queda restringido a un cambio lineal en el tiempo, sino caracterizado por un solo máximo y un solo mínimo en los puntos finales de la serie.

8. Vacilación climática: "fluctuación" en la cual la variable climática tiende a permanecer alternativamente en torno a dos (o más) valores medios, y a pasar de un valor medio a otro a intervalos regulares o irregulares (por oposición a "oscilación").

9. Variación climática: una "fluctuación" o una componente de ésta cuya escala temporal característica es lo suficientemente amplia como para conducir a una inconstancia apreciable de las medias (normales) sucesivas de las variables, calculadas para un período de treinta años. ${ }^{1}$

La realidad, sin embargo, es que desde el punto de vista de la Climatología, la expresión "cambio climático" se ha utilizado y se utiliza en el sentido de una discontinuidad o ruptura más bien brusca y permanente, de las condiciones existentes hacia otras diferentes, es decir, desde unos valores característicos de la serie a otros valores. Consecuentemente, "el cambio climático implicaría el paso de un estado climático a otro", y podría definirse, por la existencia de una modificación relevante en alguno de sus valores, como podría ser, por ejemplo, la media de un parámetro, su variabilidad o ambas simultáneamente, y que tendrían gran permanencia en el tiempo.

Esta ha sido la definición que tradicionalmente se ha hecho del cambio climático en los estudios climatológicos, lo cual implica que se definiría básicamente por su magnitud tanto como por su dimensión espacial y temporal, ya que ambas tienen un papel importante en su definición, porque el mismo suele ir asociado a permanencia (ante su larga duración) y a un extenso ámbito espacial, y con este significado fue utilizado hasta mediados de los años 1970. La confusión de expresiones y conceptos es un hecho que surge durante los últimos años, debido al mal uso y abuso que se hizo de la expresión cambio climático, fundamentalmente por los no especialistas en el tema, ya que desde la Climatología, los conceptos siempre han sido claros y utilizados con claridad. Teniendo en cuenta estas consideraciones, entendemos por:

- Cambio climático: cuando la variación en alguno de los componentes del sistema es lo suficientemente importante como para alterar su equilibrio, dando lugar a un equilibrio nuevo tras un período de transición entre ambos. Ello supone que la "anomalía" en un componente ha sido lo suficientemente importante como para rebasar el umbral de estabilidad del sistema; esta anomalía influye a su vez en otro componente del sistema, que su vez repercute en otro, y así sucesivamente, en consecuencia el sistema no tiene ya una dinámica propia de un sistema estacionario, sino de un sistema que cambia, que experimenta una tendencia clara hacia otra situación, pudiendo llegar a alcanzar un nuevo equilibrio en un momento dado.

Así pues, un cambio climático, por oposición a una mera anomalía o fluctuación, presenta ciertos caracteres que lo definen y que pueden sintetizarse en tres: 
- el sistema no vuelve ya a su estado anterior, sino que evoluciona hacia un nuevo estado, diferente al anterior, hasta alcanzar un nuevo equilibrio,

- el cambio afecta a todo el sistema en virtud de las interrelaciones que se establecen entre todos sus componentes,

- las anomalías locales no serán ya compensatorias y mantenedoras del equilibrio global, porque ahora lo que se está produciendo es, precisamente, la ruptura del equilibrio.

Queda, por lo tanto, circunscrito a aquellos cambios de gran magnitud (de 6 a $10^{\circ} \mathrm{C}$ más elevados o inferiores a los actuales) y gran escala temporal y espacial (con una duración de al menos miles de años y a nivel planetario o hemisférico), donde el cambio es la norma y no la excepción (BRUNIARD, 1999), mientras que entendemos por:

- Oscilación climática, aquellas variaciones que abarcan una escala temporal de varias décadas a siglos y milenios.

- Variación climática, cuando las fluctuaciones son lo suficientemente largas como para influir en una media de 30 años.

- Fluctuación climática, las diferencias de corto período de duración (por ejemplo estacional, interanual, decenal).

Para mayor claridad en los conceptos, se presenta en la Figura 1, algunas formas de fluctuaciones y variaciones climáticas relacionadas con la variabilidad climática, que es necesario tener en cuenta a la hora de evaluar la misma.

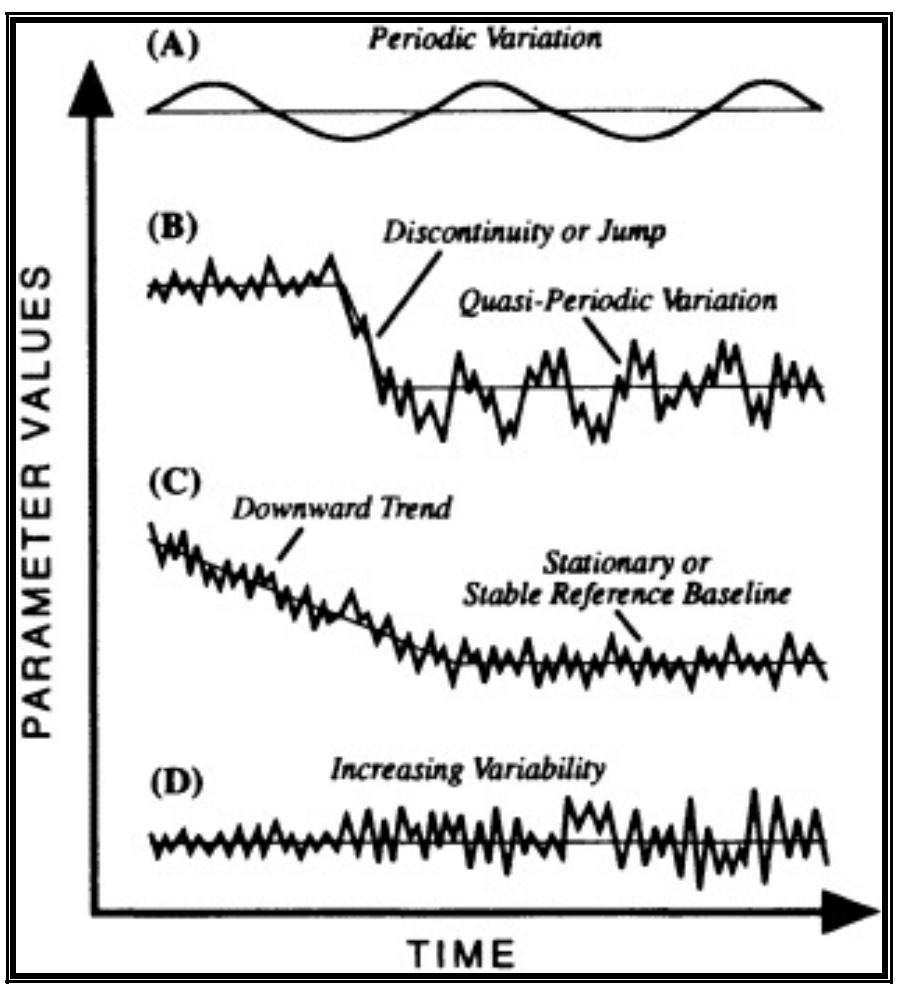

FIGURA 1. Tipos de fluctuaciones y variaciones climáticas. Tomado de: Natural Climate Variability on Decade to Century Time Scale (1999). National Academic Press, Washington, p. 7.

A. La variabilidad climática puede presentar Variaciones o Fluctuaciones Periódicas que son similares en su naturaleza, al ciclo diario o anual, pero en este caso es un ciclo que tiende a durar de 10 a 100 años de longitud.

B. El clima puede también experimentar una repentina Discontinuidad o Salto desde su estado corriente a un estadio diferente, caracterizado por significativas condiciones de enfriamiento o calentamiento.

C. Puede mostrar una Tendencia descendente (o ascendente) continua antes de alcanzar un nuevo estado de referencia o nivel de base estable. 
D. Por último, el clima puede mantener lo que aparentemente es un estado tranquilo o estable cuando se lo caracteriza por una variable específica como la temperatura media anual, pero las variaciones en algún otro parámetro como la temperatura estacional, la amplitud diurna de la temperatura, la frecuencia de tormentas, etc., pueden indicar que ha tenido lugar un cambio significativo a través de un Aumento en la Variabilidad del mismo.

Se puede concluir, por lo tanto, que el problema principal radica en el sentido que se le da actualmente a la expresión "cambio climático", indicando con ella todas las formas de inconstancia climática (esto es, cualquier diferencia entre las valores a largo plazo de los elementos meteorológicos calculados para distintos períodos pero respecto a la misma zona), con independencia de sus causas físicas. De esta manera, por desconocimiento y mal manejo del término, desde los medios periodísticos y revistas de difusión se le asigna el carácter de cambio climático a cualquier fenómeno o evento poco común o que posea una magnitud mayor a la conocida o registrada hasta ese momento, cuando probablemente el fenómeno sólo sea poco frecuente, actitud que sólo genera confusiones y alarma entre la población, mientras que desde el punto de vista climatológico la expresión tiene un significado bien preciso y diferente.

\section{Fluctuaciones y variabilidad de la temperatura media anual y estacional en el Norte Argentino}

Realizadas estas aclaraciones, se va a analizar la marcha o comportamiento de la temperatura media anual y media estacional en las localidades del Norte de nuestro país, puesto que dentro de la "hipótesis de cambio climático" de la que tanto se habla, la temperatura es el elemento del clima al que más referencia se hace en relación con el denominado "calentamiento global". La temperatura, por otra parte, es el elemento que probablemente influye más que cualquier otro sobre nuestro nivel de confort diario y, porque desde una perspectiva más amplia, la distribución de la temperatura global es la "máquina" que maneja el sistema climático de la Tierra. Tal como lo expresa Fernández García (1996), "la temperatura controla los patrones de la circulación general de la atmósfera, que a su vez influyen en la precipitación y en la evaporación, las trayectorias y la intensidad de las tormentas entre otros patrones climáticos de gran escala. Consecuentemente, mientras el impacto directo del cambio de la temperatura en la sociedad es considerable, su influencia en otros importantes atributos climáticos, son enormes".

En la mayoría de los estudios e investigaciones llevados a cabo por especialistas de distintas partes del mundo, se hace referencia a que las mediciones y registros existentes, muestran que la temperatura del aire cerca de la superficie posee un sostenido y progresivo aumento desde finales del siglo XIX y principios del XX. Las mejores estimaciones sugieren que el aumento o calentamiento total ha sido de alrededor de $0.5^{\circ} / 0.6^{\circ} \mathrm{C}$. Sin embargo, muchos investigadores también han cuestionado lo adecuado de estas estimaciones, por la relativa escasez de las mediciones globales a través del siglo, y porque el aumento no se ha producido al mismo ritmo, con la misma intensidad ni de manera uniforme en todo el planeta. Algunas regiones por el contrario, no registran el aumento global de la temperatura, sino una disminución de los valores térmicos, tales como: el centro y norte de Canadá, el sureste de Estados Unidos, el noroeste del Atlántico Norte, el centro de Africa, Tíbet, Bolivia. $^{2}$

Nuestro país cuenta con la información meteorológica necesaria para detectar fluctuaciones a corto plazo y mediano plazo, ya que las series más extensas que se pueden conseguir, abarcan en la mayoría de las estaciones, el período 1931/2005. Cuenta al mismo tiempo, con una serie de inconvenientes, comunes a nivel mundial, relacionados con el emplazamiento de las estaciones, la recolección de los datos, cambios en los instrumentos, etc. Las estaciones meteorológicas existentes en el norte de Argentina, generalmente emplazadas en los aeropuertos y aeródromos locales, se muestran en la Figura 2. Si bien cada punto localizado representa una estación, no todas contienen el mismo período de información, e incluso muchas de ellas han sido levantadas a partir de 1960, de manera que sólo contamos con información para el período de estudio de las estaciones cuyos nombres figuran en el mapa.

Dicha información, por otra parte, no siempre está completa, tales los casos de Las Lomitas (sólo cuenta con información desde 1951), Tucumán y Rivadavia (cuyas serie se cortan en 1990), y La Quiaca (que posee datos hasta el 2005 pero la década 1991/2000 está muy incompleta). No obstante, se han considerado estas dos últimas estaciones para el análisis, a las que se ha agregado la estación La Rioja para completar el panorama del norte de nuestro país. 


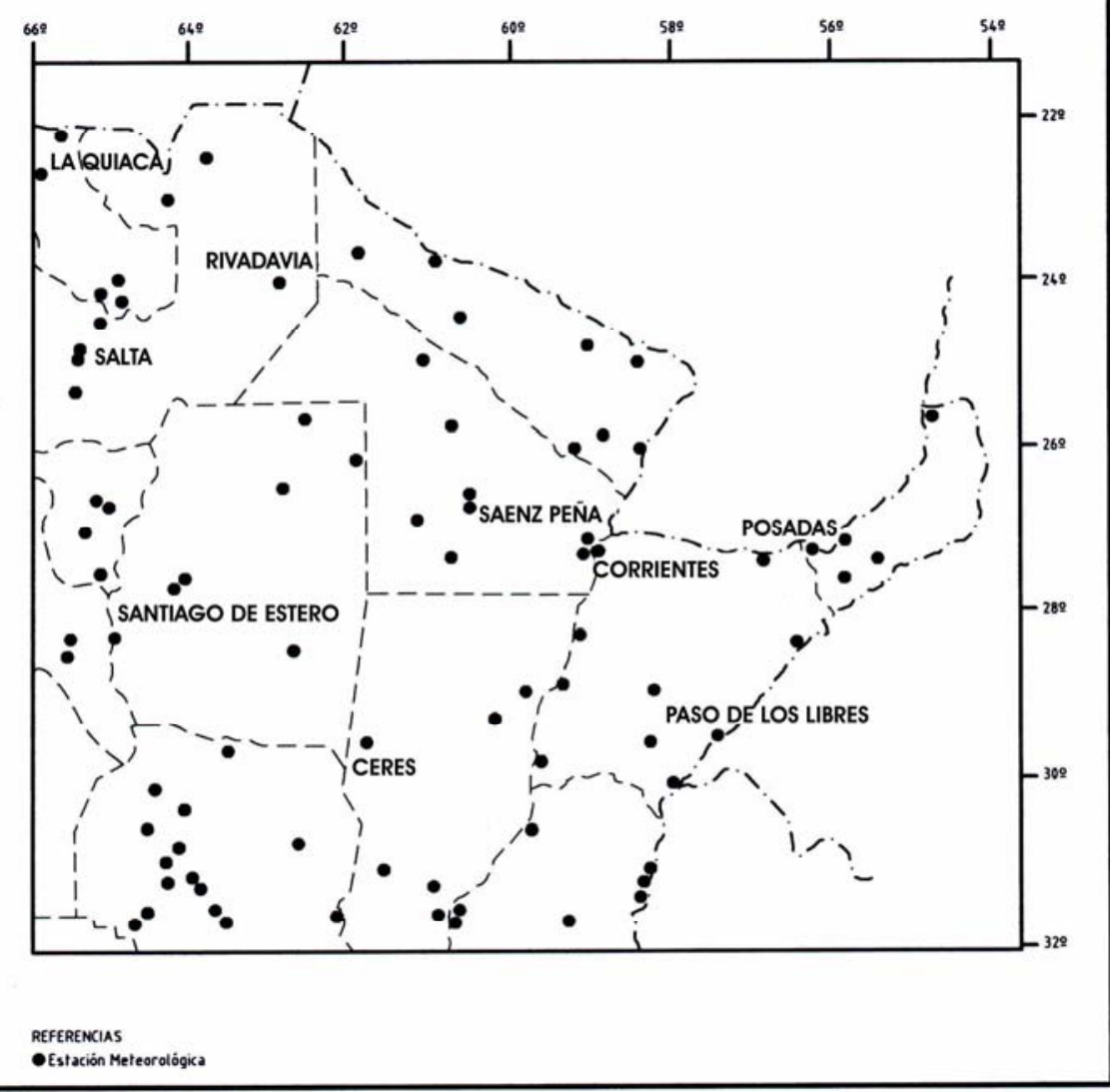

\section{FIGURA 2. Localización Estaciones Meteorológicas del Norte de Argentina}

Las series analizadas corresponden a las proporcionadas por el sitio web de la NASA-GISS (Goddard Institute for Space Studies) - Surface Temperature Análisis - Station Data, con la excepción de la estación Sáenz Peña, cuyos datos fueron proporcionados por el Ing. Angeloni, y que pertenecen a los registros del INTA de dicha localidad. ${ }^{3}$

En cuanto a los gráficos realizados, cabe aclarar que en cada una de las representaciones se ha mantenido constante la escala a efectos de una mejor comparación (las diferencias que se pueden detectar en los valores corresponden a las variaciones de la temperatura de cada estación). En el caso de:

- la temperatura media anual, la equidistancia seleccionada fue de $4^{\circ} \mathrm{C}$, en

- la temperatura media estacional, como las diferencias interestacionales son mayores que las anuales, la equidistancia se aumentó a $6^{\circ} \mathrm{C}$, en

- los promedios suavizados y las desviaciones desde la normal, se procedió de la misma manera.

\section{a. Las Fluctuaciones Térmicas Anuales}

Al representar los valores del período comprendido entre 1970 y 2005, se advierte fácilmente que la temperatura media anual presenta una tendencia al aumento en la mayoría de las estaciones, salvo en Sáenz Peña, Paso de los Libres y Ceres, en que la misma es descendente. La tendencia se detecta más claramente cuando a los gráficos se les agrega la recta de tendencia lineal (o regresión lineal por mínimos cuadrados), representada en los gráficos con trazo azul. ${ }^{4}$ 
TEMPERATURA MEDIA ANUAL (en ${ }^{\circ} \mathrm{C}$ ) - PERIODO 1970/2005
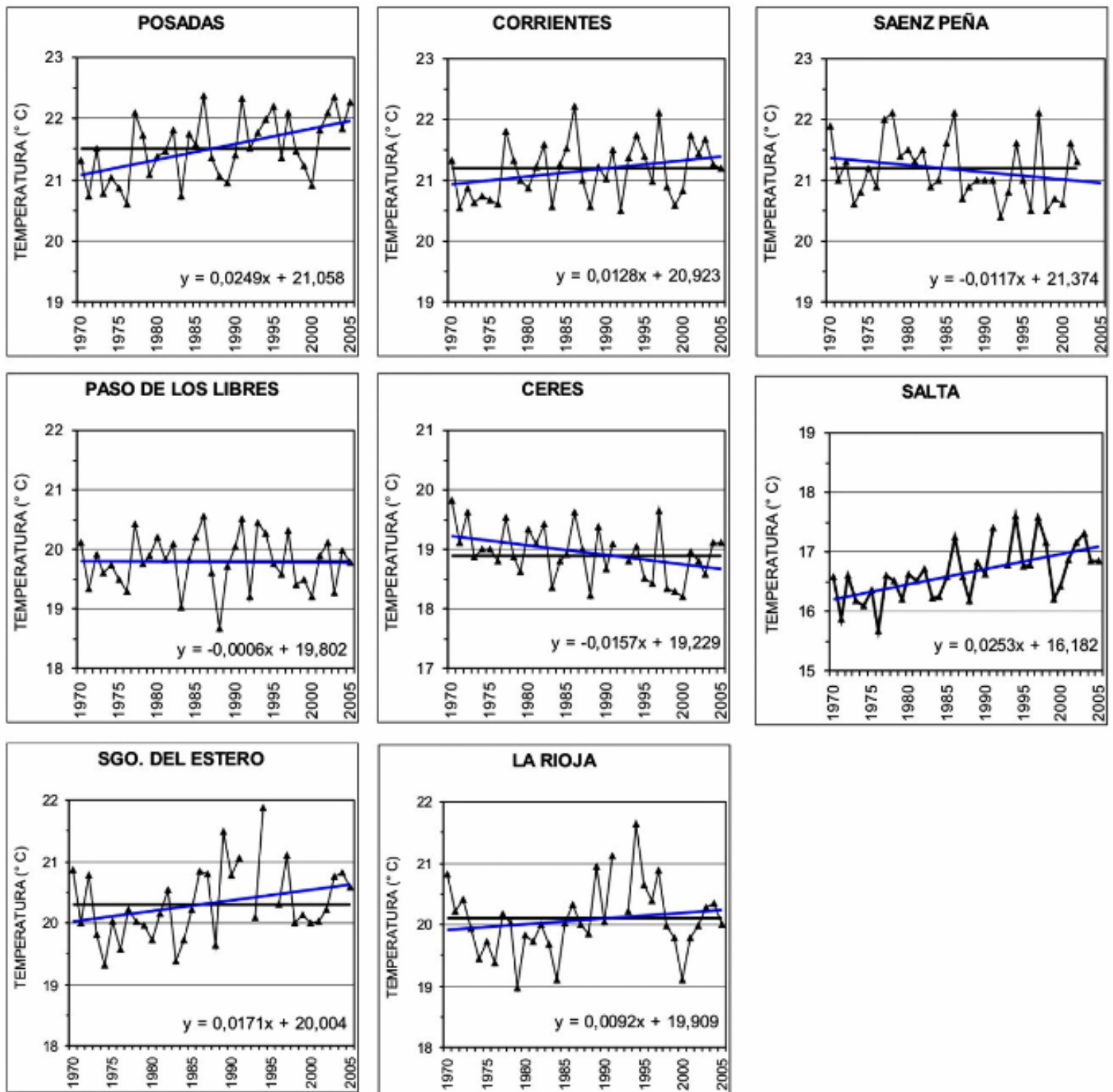

FUENTE: NASA-GISS (Goddard Institute for Space Studies)

\section{FIGURA 3. Temperatura Media Anual - Período 1970/2005}

Pero, si en vez de representar los últimos 35 años representamos la serie completa de datos, que comprende el período 1931/2005, advertimos rápidamente que el comportamiento de la temperatura media anual presenta algunos cambios en cuanto a la tendencia lineal, pues ahora es descendente en la mayoría de las estaciones, siendo las excepciones sólo Posadas y La Rioja, que mantienen sus temperaturas medias anuales en ascenso, aunque en menor medida. ${ }^{5}$ 
TEMPERATURA MEDIA ANUAL (en ${ }^{\circ} \mathrm{C}$ ) - PERIODO 1931/2005
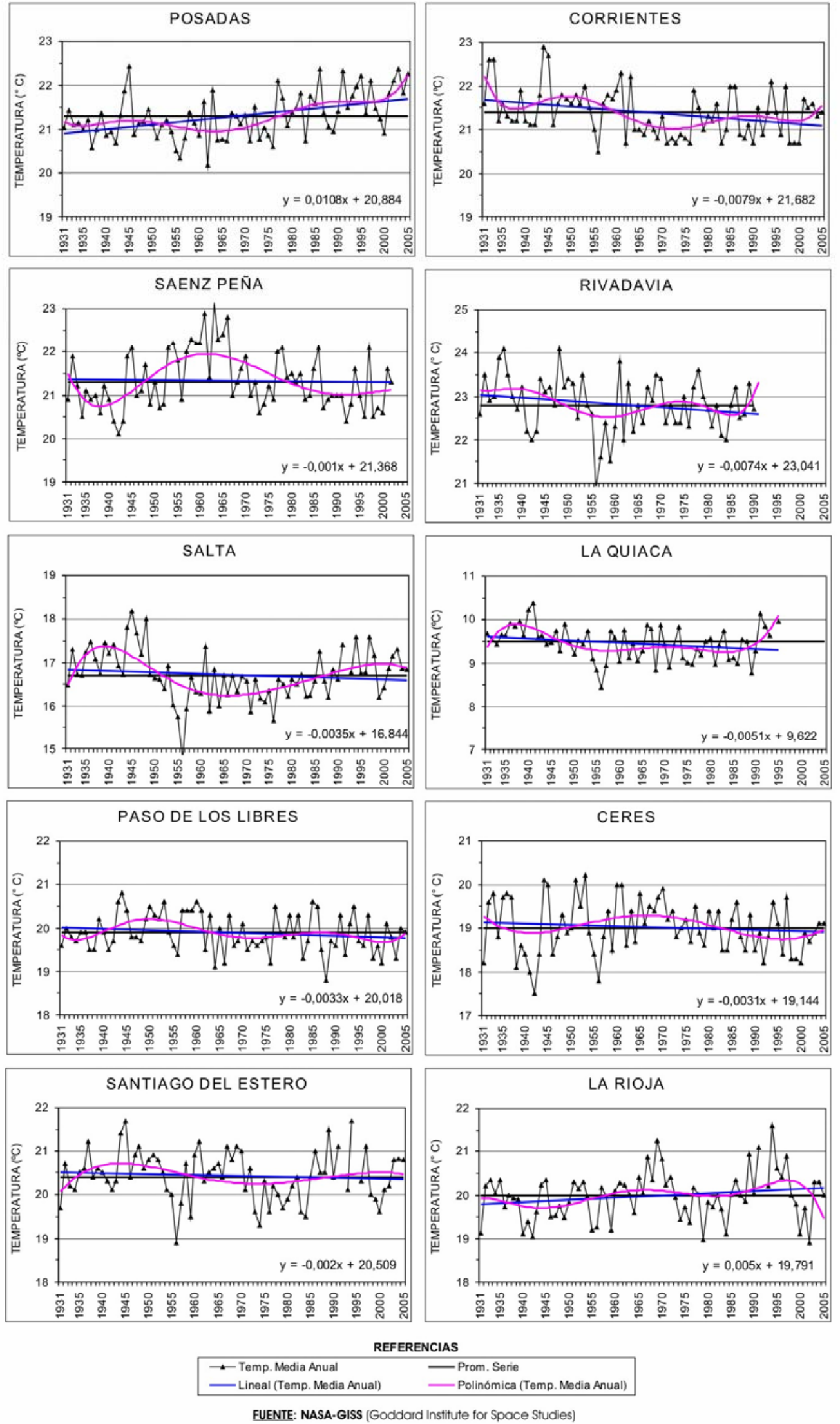
FIGURA 4. Temperatura Media Anual - Período 1931/2005.

En cuanto a los valores de la pendiente de la recta de los dos períodos considerados, los mismos se consignan en la tabla 1.

\section{TABLA 1. Comparación de los valores de las pendientes de la recta - Períodos 1970/2005 y $1931 / 2005$}

\begin{tabular}{|l|c|c|}
\hline \multicolumn{1}{|c|}{ ESTACION } & $\mathbf{1 9 7 0 / 2 0 0 5}$ & $\mathbf{1 9 3 1 / 2 0 0 5}$ \\
\hline Posadas & 0,0249 & 0.0108 \\
\hline Corrientes & 0,0128 & -0.0079 \\
\hline Sáenz Peña & $-0,0117$ & -0.0010 \\
\hline Paso de los Libres & $-0,0006$ & -0.0033 \\
\hline Ceres & $-0,0157$ & -0.0031 \\
\hline Salta & 0,0253 & -0.0035 \\
\hline Santiago del Estero & 0,0171 & -0.0020 \\
\hline La Rioja & 0,0092 & 0.0050 \\
\hline
\end{tabular}

Se destaca el caso de Posadas, en que la pendiente es bastante marcada, y en la que el sostenido aumento se inicia hacia 1975, siendo la única estación que presenta esta característica en todo el Norte argentino.

Como las diferencias interanuales, no se ajustan perfectamente a las condiciones que debe reunir una recta de tendencia lineal, se ha incluido en los gráficos, la curva de tendencia polinómica, representada con trazo de color fucsia. Estas curvas nos indican que el comportamiento de la temperatura no es uniforme, temporal ni espacialmente, sino que presenta fluctuaciones que señalan décadas o períodos más cálidos que alternan con otros más fríos o frescos, es decir, fluctuaciones que oscilan alrededor del valor medio, representado en los gráficos con trazo negro. ${ }^{6}$

El análisis de los gráficos permite, al mismo tiempo, identificar algunas similitudes regionales, como por ejemplo, que el patrón que siguen las ondulaciones de la tendencia polinómica, independientemente de la tendencia lineal, es similar:

- en las estaciones localizadas en el oriente del área de estudio (Posadas, Corrientes y Paso de los Libres, tienen un patrón similar, si bien se detectan algunas diferencias, tales como la marcada tendencia positiva de Posadas a partir de 1975 y la mayor suavidad de la curva de Paso de los Libres),

- en las estaciones del centro del territorio, es notable la semejanza entre Sáenz Peña y Ceres, si bien en esta última la tendencia es mucho más suave, finalmente,

- el comportamiento se invierte o es relativamente opuesto en las estaciones del oeste: Rivadavia, Salta, La Quiaca, Santiago del Estero y La Rioja.

Se puede destacar también que, si bien la tendencia lineal es negativa en la mayoría de los casos, la tendencia polinómica indica un aumento de la temperatura a partir de los años 1995/2000 en las estaciones del oriente y centro del área de estudio, mientras que en Salta, Santiago del Estero y La Rioja, la tendencia polinómica revela un descenso de la temperatura, a partir aproximadamente, de la misma época.

Otra característica que resalta en el comportamiento de la temperatura es que, en general, contrariamente a lo registrado a nivel global y hemisférico, los años más cálidos fueron los de 1944 y 1945 (Corrientes, Paso de los Libre, Salta y Santiago del Estero), 1953 (Ceres), 1963 (Sáenz Peña, 1994 (La Rioja) y 2003 (Posadas). ${ }^{7}$

Los años más fríos, también en general se registraron en 1956 (Salta Santiago del Estero, Posadas y Corrientes), 1942 (Sáenz Peña y Ceres), 1978 (La Rioja) y 1988 (Paso de los Libres). ${ }^{8}$

Los promedios anuales decenales, consignados en la Tabla 2, cuyos valores más altos y bajos principales y secundarios se han resaltado con color rojo y azul respectivamente, permiten expresar algunas conclusiones. 
TABLA 2. Temperatura Media Anual por Década - Período 1931/1990

\begin{tabular}{|l|c|c|c|c|c|c|c|c|}
\hline \multicolumn{1}{|c|}{ ESTACION } & $\mathbf{1 9 3 1 / 4 0}$ & $\mathbf{1 9 4 1 / 5 0}$ & $\mathbf{1 9 5 1 / 6 0}$ & $\mathbf{1 9 6 1 / 7 0}$ & $\mathbf{1 9 7 1 / 8 0}$ & $\mathbf{1 9 8 1 / 9 0}$ & $\mathbf{1 9 9 1 / 0 0}$ & $\begin{array}{c}\text { PROM. } \\
\text { SERIE }\end{array}$ \\
\hline Salta & 17,0 & $\mathbf{1 7 , 3}$ & $\mathbf{1 6 , 2}$ & 16,5 & 16,3 & $\mathbf{1 6 , 6}$ & $\mathbf{1 6 , 9}$ & $\mathbf{1 6 , 7}$ \\
\hline Corrientes & 21,6 & $\mathbf{2 1 , 7}$ & 21,5 & 21,2 & $\mathbf{2 0 , 9}$ & $\mathbf{2 1 , 2}$ & 21,2 & $\mathbf{2 1 , 3}$ \\
\hline Sgo, del Estero & 20,4 & $\mathbf{2 0 , 8}$ & 20,2 & $\mathbf{2 0 , 8}$ & $\mathbf{1 9 , 9}$ & 20,4 & 20,4 & $\mathbf{2 0 , 4}$ \\
\hline P. de los Libres & $\mathbf{1 9 , 7}$ & 20,1 & $\mathbf{2 0 , 2}$ & 19,8 & 19,8 & 19,8 & 19,8 & $\mathbf{1 9 , 9}$ \\
\hline Sáenz Peña & 21,0 & 21,1 & 21,7 & $\mathbf{2 2 , 1}$ & 21,3 & 21,2 & $\mathbf{2 0 , 9}$ & $\mathbf{2 1 , 3}$ \\
\hline Ceres & 19,1 & 18,8 & 19,2 & $\mathbf{1 9 , 4}$ & 19,1 & 18,9 & $\mathbf{1 8 , 7}$ & $\mathbf{1 9 , 0}$ \\
\hline La Rioja & 19,9 & $\mathbf{1 9 , 7}$ & 19,9 & $\mathbf{2 0 , 4}$ & 19,8 & 20,0 & $\mathbf{2 0 , 4}$ & $\mathbf{2 0 , 0}$ \\
\hline Posadas & 21,1 & 21,3 & $\mathbf{2 0 , 9}$ & 21,1 & 21,2 & 21,4 & $\mathbf{2 1 , 7}$ & $\mathbf{2 1 , 2}$ \\
\hline La Quiaca & $\mathbf{9 , 6}$ & 9,5 & $\mathbf{9 , 2}$ & 9,5 & 9,2 & 9,2 & s/d & s/d \\
\hline Rivadavia & $\mathbf{2 3 , 2}$ & 22,9 & $\mathbf{2 2 , 3}$ & 22,9 & $\mathbf{2 2 , 8}$ & 22,6 & s/d & s/d \\
\hline
\end{tabular}

FUENTE: cuadro elaborado a partir de los datos proporcionados por el sitio web NASA-GISS (Goddard Institute for Space Studies)

- Durante la primera mitad de la serie (décadas de 1931 a 1970) se encuentran los registros térmicos más altos (1931/40 y 1941/50 para Salta, Corrientes, Santiago del Estero, La Quiaca y Rivadavia; 1951/60 y 1961/70 para Sáenz Peña y Ceres; 1951/60 para Paso de los Libres, entre otras), mientras que,

- Durante la segunda mitad de la misma predominan, en general, las décadas más frías (1971/80 para Corrientes y Santiago del Estero; 1991/00 para Sáenz Peña y Ceres, etc.),

- Las excepciones la constituyen las estaciones Posadas y La Rioja, si bien en esta última el mismo valor se registró también en la década 1961/70.

\section{b. Las Fluctuaciones Térmicas Estacionales}

Características similares se detectan en las fluctuaciones de la temperatura media estacional. En este caso, se han calculado los valores considerando las estaciones astronómicas, a efectos de poder comparar con los promedios globales y hemisféricos, y ver el grado de correlación existente entre ambos.

Temperatura Media de Verano. El verano comprende los meses de Diciembre-Enero-Febrero (el mes de Diciembre corresponde al año anterior, ya que el verano del Hemisferio Sur está cortado, es decir que el verano 1931/32 comprende los meses de Diciembre de 1931, Enero y Febrero de 1932, tal como figura en las representaciones gráficas).

La estación de verano, al igual que la temperatura media anual, presenta un comportamiento común o similar en las localidades del norte, tal como se observa en la Figura 5. 
TEMPERATURA MEDIA ANUAL (en ${ }^{\circ}$ C) - PERIODO 1930/31 - 2004/2005
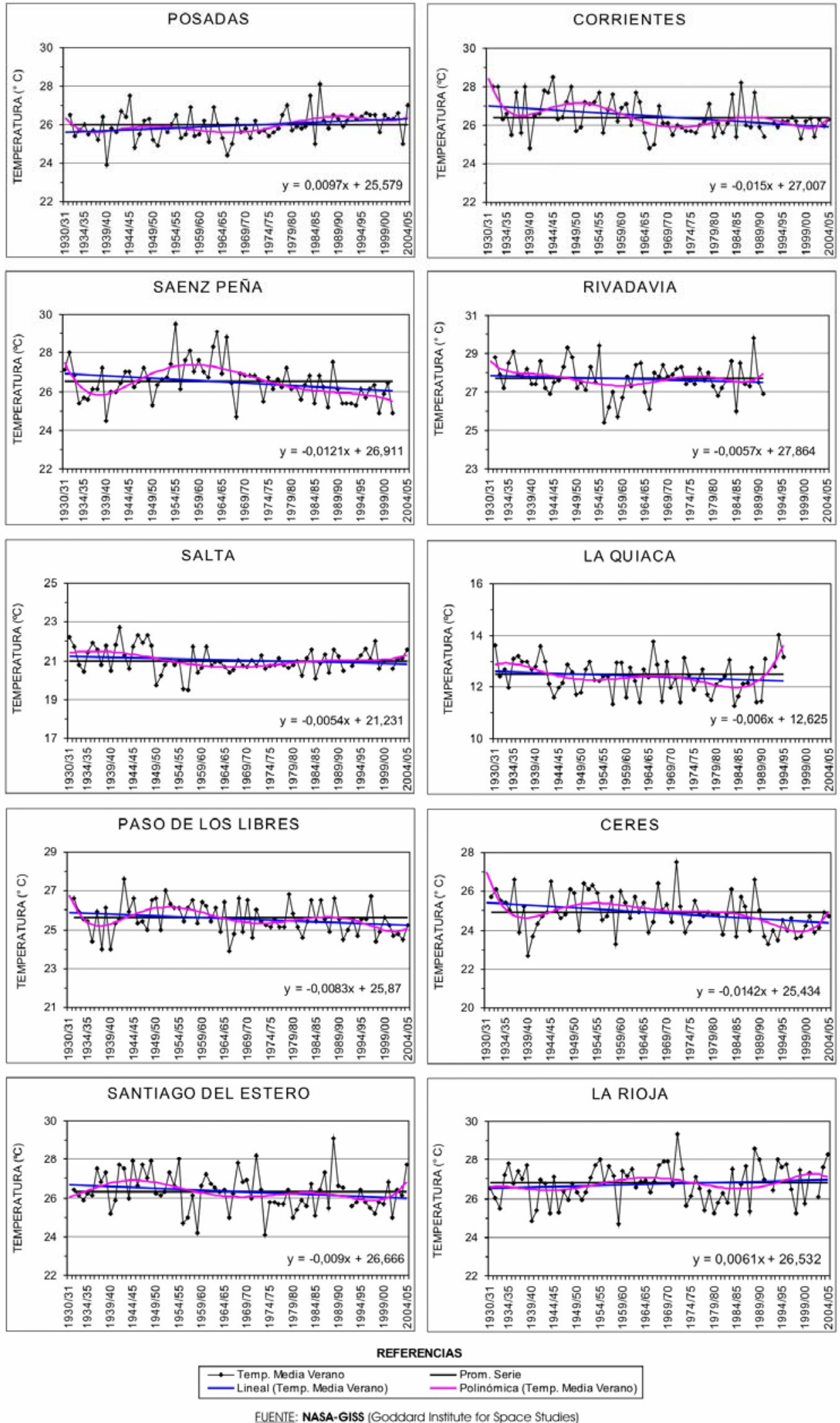


\section{FIGURA 5. Temperatura Media de Verano - Período 1931/32 - 2004/2005}

Las características más destacables, en cuanto a la tendencia lineal, es que es negativa (descendente) en la mayoría de las estaciones; los descensos más marcados se registran en Sáenz Peña y Ceres. Es positiva sólo en Posadas y La Rioja, en que es suavemente ascendente.

En cuanto a la tendencia polinómica, se puede advertir que su trazado, en general es muy suave, especialmente en Posadas, Rivadavia, Salta y La Rioja y es algo más marcado en el resto de las estaciones, destacándose Sáenz Peña.

En este caso también se pueden detectan patrones o similitudes en el comportamiento de la temperatura media de verano entre las estaciones, que a grandes rasgos repiten las similitudes advertidas en la temperatura media anual, Por ejemplo:

- Posadas, Corrientes y Paso de los Libres (aunque poseen distinta intensidad),

- Sáenz Peña y Ceres (si bien en Sáenz Peña es mucho más marcada),

- Salta y Santiago del Estero (Salta es mucho más suave), mientras que La Rioja presenta características propias.

Respecto de las diferencias interestacionales, las menores se localizan en Posadas y Paso de los Libres, Salta y, en menor medida y en diferentes períodos en Corrientes, mientras que las más marcadas se encuentran en Sáenz Peña, Ceres, Rivadavia, Santiago del Estero y La Rioja. Las causas se deberían a la localización geográfica de las estaciones, en relación con la circulación atmosférica, la influencia marítima/continental y la altura.

Otra característica a destacar es el descenso bastante marcado que presentan los valores a partir de $1985 / 90$ y que se mantiene hasta aproximadamente el año 2000, en que nuevamente asciende la temperatura, siendo la excepción en este caso, la estación La Rioja.

El análisis de los valores medios anuales decenales de verano, consignados en la Tabla 3, cuyos valores más altos y bajos principales y secundarios se han resaltado con color rojo y azul respectivamente, permite manifestar algunas conclusiones.

TABLA 3. TEMPERATURA MEDIA DECENAL DE VERANO - PERIODO 1931/1990

\begin{tabular}{|l|c|c|c|c|c|c|c|c|}
\hline \multicolumn{1}{|c|}{ ESTACION } & $\mathbf{1 9 3 1 / 4 0}$ & $\mathbf{1 9 4 1 / 5 0}$ & $\mathbf{1 9 5 1 / 6 0}$ & $\mathbf{1 9 6 1 / 7 0}$ & $\mathbf{1 9 7 1 / 8 0}$ & $\mathbf{1 9 8 1 / 9 0}$ & $\mathbf{1 9 9 1 / 0 0}$ & $\begin{array}{c}\text { PROM. } \\
\text { SERIE }\end{array}$ \\
\hline Salta & 21,3 & $\mathbf{2 1 , 6}$ & $\mathbf{2 0 , 6}$ & 20,8 & 20,8 & 20,9 & 21,1 & $\mathbf{2 1 , 0}$ \\
\hline Corrientes & 26,7 & $\mathbf{2 7 , 1}$ & 26,8 & 26,3 & $\mathbf{2 5 , 9}$ & 26,4 & 26,1 & $\mathbf{2 6 , 5}$ \\
\hline Sgo. del Estero & 26,4 & $\mathbf{2 7 , 0}$ & 26,1 & 26,6 & 25,9 & 26,4 & $\mathbf{2 5 , 8}$ & $\mathbf{2 6 , 3}$ \\
\hline P. de los Libres & 25,3 & $\mathbf{2 6 , 0}$ & $\mathbf{2 6 , 0}$ & 25,6 & 25,5 & 25,6 & $\mathbf{2 5 , 2}$ & $\mathbf{2 5 , 6}$ \\
\hline Sáenz Peña & 26,3 & 26,4 & $\mathbf{2 7 , 3}$ & 27,2 & 26,5 & 26,2 & $\mathbf{2 5 , 7}$ & $\mathbf{2 6 , 5}$ \\
\hline Ceres & 25,1 & 25,1 & $\mathbf{2 5 , 3}$ & 25,1 & 25,0 & 24,9 & $\mathbf{2 4 , 0}$ & $\mathbf{2 4 , 9}$ \\
\hline La Rioja & 26,7 & $\mathbf{2 6 , 2}$ & 26,9 & $\mathbf{2 7 , 2}$ & 26,6 & 26,7 & 26,9 & $\mathbf{2 6 , 7}$ \\
\hline Posadas & $\mathbf{2 5 , 6}$ & 26,0 & 25,8 & 25,7 & 25,9 & $\mathbf{2 6 , 3}$ & $\mathbf{2 6 , 3}$ & $\mathbf{2 5 , 9}$ \\
\hline La Quiaca & $\mathbf{1 2 , 8}$ & 12,3 & 12.4 & 12.5 & 12,1 & 12,1 & $\mathrm{~s} / \mathrm{d}$ & $\mathbf{s} / \mathbf{d}$ \\
\hline Rivadavia & $\mathbf{2 8 , 1}$ & 27,9 & $\mathbf{2 7 , 2}$ & 27,7 & 27,8 & 27,6 & s/d & s/d \\
\hline
\end{tabular}

FUENTE: cuadro elaborado a partir de los datos proporcionados por el sitio web NASA-GISS (Goddard Institute for Space Studies)

- Los veranos más cálidos se concentran en la primera mitad de la serie, en las décadas de 1941/50 (Corrientes, Salta, Paso de los Libre y Santiago del Estero) y 1951/60 (Sáenz Peña, Ceres, La Rioja), algunos de cuyos registros no han sido superados hasta la actualidad,

- Los veranos más frescos, por el contrario, se encuentran en la segunda mitad de la serie, especialmente en Santiago del Estero, Paso de los Libres, Sáenz Peña y Ceres.

- nuevamente Posadas y La Rioja son las excepciones.

Temperatura Media de Otoño. El otoño, que comprende los valores medios de Marzo-Abril-Mayo, se caracteriza por presentar mayor diversidad en cuanto a las tendencias lineales, que son positivas en 
Revista Geográfica Digital. IGUNNE. Facultad de Humanidades. UNNE. Año 3- No 6 Julio - Diciembre 2006. ISSN 1668-5180. Resistencia, Chaco

Posadas, Sáenz Peña, Ceres, Santiago del Estero y La Rioja, y negativas en el resto de las estaciones, pero con pendientes muy suaves en ambos casos, tal como se observa en la figura a continuación. 
TEMPERATURA MEDIA DE OTOÑO (en ${ }^{\circ} \mathrm{C}$ ) - PERIODO 1931/2005
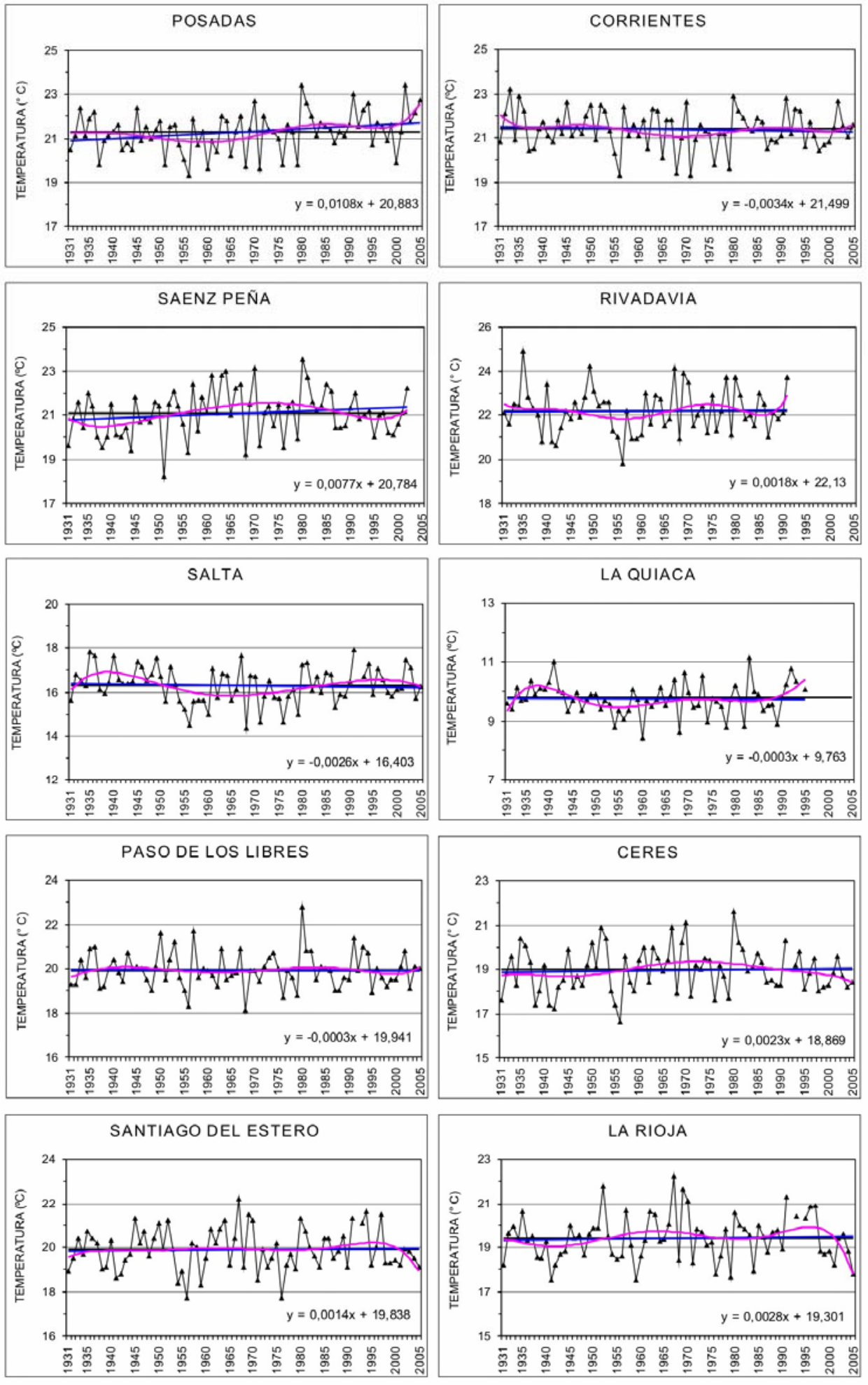

\section{REFERENCIAS}

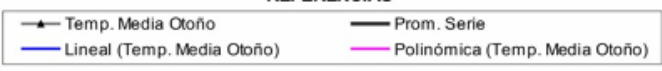

FUENTE: NASA-GISS (Goddard Institute for Space Studies) 


\section{FIGURA 6. Temperatura Media de Otoño - Período 1931/2005}

En general se puede advertir que la temperatura media de otoño posee patrones similares a los ya enunciados, en el comportamiento de la temperatura media anual. Independientemente de las tendencias lineales, las tendencias polinómicas, durante los últimos años de la serie son ascendentes en Posadas, Corrientes, Sáenz Peña, descendente en Salta, Ceres, Santiago del Estero y La Rioja, mientras que se mantiene prácticamente recta en Paso de los Libres.

Debe señalarse que las fluctuaciones interanuales son algo más marcadas que las de verano en todas la localidades, destacándose las de las estaciones del centro y occidente del área de estudio, con la excepción de Salta y La Quiaca, debido a las causas ya mencionadas.

Al analizar los valores medios anuales decenales, tal como se advierte en la tabla a continuación (cuyos valores más altos y bajos se han resaltado con color rojo y azul respectivamente), se desprende que:

TABLA 4. TEMPERATURA MEDIA DECENAL DE OTOÑO - PERIODO 1931/1990

\begin{tabular}{|l|c|c|c|c|c|c|c|c|}
\hline \multicolumn{1}{|c|}{ ESTACION } & $\mathbf{1 9 3 1 / 4 0}$ & $\mathbf{1 9 4 1 / 5 0}$ & $\mathbf{1 9 5 1 / 6 0}$ & $\mathbf{1 9 6 1 / 7 0}$ & $\mathbf{1 9 7 1 / 8 0}$ & $\mathbf{1 9 8 1 / 9 0}$ & $\mathbf{1 9 9 1 / 0 0}$ & $\begin{array}{c}\text { PROM. } \\
\text { SERIE }\end{array}$ \\
\hline Salta & 16,7 & $\mathbf{1 6 , 8}$ & $\mathbf{1 5 , 6}$ & $\mathbf{1 6 , 3}$ & 15,7 & 16,3 & 16,6 & $\mathbf{1 6 , 3}$ \\
\hline Corrientes & $\mathbf{2 1 , 6}$ & $\mathbf{2 1 , 6}$ & $\mathbf{2 1 , 3}$ & $\mathbf{2 1 , 3}$ & $\mathbf{2 0 , 9}$ & $\mathbf{2 1 , 4}$ & 21,2 & $\mathbf{2 1 , 3}$ \\
\hline P. de los Libres & 19,9 & $\mathbf{2 0 , 0}$ & 19,9 & 19,8 & $\mathbf{2 0 , 0}$ & 19,8 & $\mathbf{2 0 , 0}$ & $\mathbf{1 9 , 9}$ \\
\hline Sgo. del Estero & 19,8 & 20,0 & $\mathbf{1 9 , 4}$ & $\mathbf{2 0 , 7}$ & $\mathbf{1 9 , 4}$ & 19,9 & 20,3 & $\mathbf{1 9 , 9}$ \\
\hline Sáenz Peña & $\mathbf{2 0 , 7}$ & $\mathbf{2 0 , 7}$ & 20,9 & $\mathbf{2 1 , 9}$ & 21,0 & 21,4 & 20,8 & $\mathbf{2 1 , 1}$ \\
\hline Ceres & 18,9 & $\mathbf{1 8 , 6}$ & 18,8 & $\mathbf{1 9 , 6}$ & 19,0 & 19,1 & 18,9 & $\mathbf{1 9 , 0}$ \\
\hline La Rioja & 19,3 & $\mathbf{1 9 , 0}$ & 19,3 & $\mathbf{2 0 , 3}$ & 19,1 & 19,4 & 20,0 & $\mathbf{1 9 , 5}$ \\
\hline Posadas & 21,2 & 21,2 & $\mathbf{2 0 , 6}$ & 21,2 & 21,1 & 21,5 & $\mathbf{2 1 , 6}$ & $\mathbf{2 1 , 2}$ \\
\hline La Quiaca & 9,9 & 9,9 & 9,3 & 9,8 & 9,6 & 9.7 & s/d & s/d \\
\hline Rivadavia & 22,6 & 22,2 & 21,5 & 22,6 & 22,2 & 22,1 & s/d & s/d \\
\hline
\end{tabular}

FUENTE: cuadro elaborado a partir de los datos proporcionados por el sitio web NASA-GISS (Goddard Institute for Space Studies)

- hay más dispersión entre los registros, en cuanto que los otoños más cálidos y más frescos no se presentan tan nítidamente agrupados en la primera o segunda mitad de la serie, como en los casos anteriores, probablemente debido a las características de la circulación atmosférica,

- los otoños más calientes están en las décadas de 1931/40 y 1941/50 en Salta, Corrientes y Paso de los Libres (posiblemente también La Quiaca y Rivadavia), y en las de 1961/70 en Saénz Peña, Ceres, Santiago del Estero y La Rioja.

Temperatura media de Invierno. El invierno comprende los meses de Junio-Julio-Agosto y se caracteriza por presentar tendencias lineales muy suaves. De las estaciones series completas, sólo Posadas presenta tendencia al ascenso en los valores térmicos invernales, mientras que en el resto de ellas es levemente descendente. 
TEMPERATURA MEDIA ANUAL DE INVIERNO (en $\left.{ }^{\circ} \mathrm{C}\right)$ - PERIODO 1931/2005
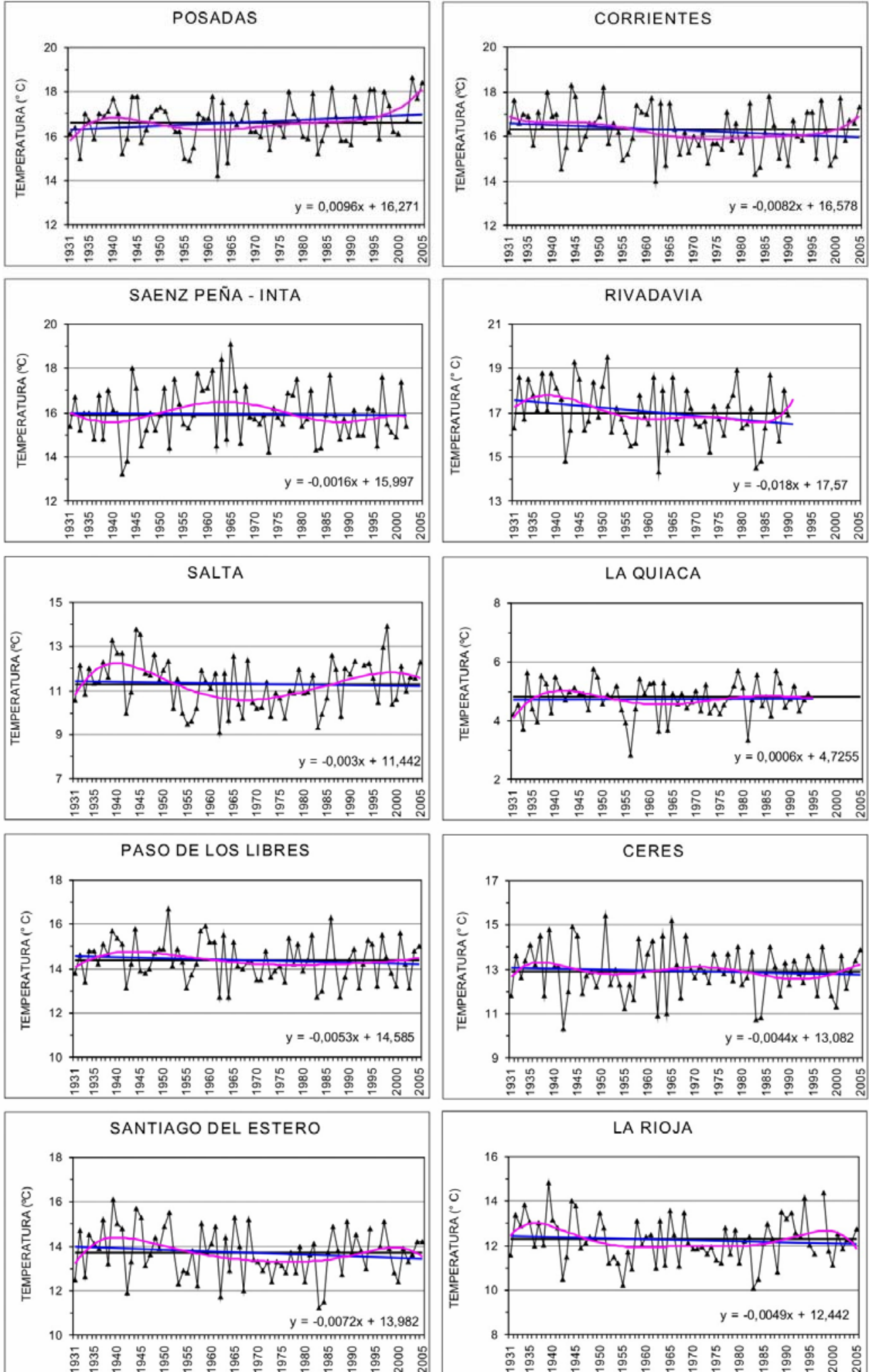

REFERENCIAS

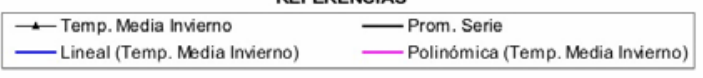

FUENIE: NASA-GISS (Goddard Institute for Space Studies) 


\section{FIGURA 7. Temperatura Media de Invierno - Período 1931/2005}

En cuanto a las tendencias polinómicas, los patrones son similares a los ya indicados en cuanto al comportamiento temporal y espacial de la temperatura media anual. Se puede destacar que, independientemente de la tendencia lineal, los últimos años de las series evidencian un aumento de los valores en las estaciones localizadas en el oriente del área de estudio, mientras que en las situadas en el occidente, se detecta un descenso de los mismos.

Se advierte también que esta estación se identifica por poseer las fluctuaciones interanuales bastante más marcadas, producto de la posición latitudinal y de la influencia del sistema circulatorio de la zona subtropical, sometida al continuo influjo de masas de aire polares y tropicales.

El análisis de los valores medios anuales decenales, tal como se advierte en la tabla 6 (cuyos valores más altos y bajos se han resaltado con color rojo y azul respectivamente), permite concluir que:

TABLA 6. TEMPERATURA MEDIA DECENAL DE INVIERNO - PERIODO 1931/1990

\begin{tabular}{|l|c|c|c|c|c|c|c|c|}
\hline \multicolumn{1}{|c|}{ ESTACION } & $\mathbf{1 9 3 1 / 4 0}$ & $\mathbf{1 9 4 1 / 5 0}$ & $\mathbf{1 9 5 1 / 6 0}$ & $\mathbf{1 9 6 1 / 7 0}$ & $\mathbf{1 9 7 1 / 8 0}$ & $\mathbf{1 9 8 1 / 9 0}$ & $\mathbf{1 9 9 1 / 0 0}$ & $\begin{array}{c}\text { PROM. } \\
\text { SERIE }\end{array}$ \\
\hline Salta & 11,8 & $\mathbf{1 2 , 1}$ & $\mathbf{1 0 , 8}$ & 10,8 & 10,8 & 11,1 & 11,8 & $\mathbf{1 1 , 3}$ \\
\hline Corrientes & $\mathbf{1 6 , 8}$ & 16,5 & 16,4 & 16,1 & $\mathbf{1 5 , 8}$ & 15,9 & 16,1 & $\mathbf{1 6 , 2}$ \\
\hline P. de los Libres & 14,6 & 14,4 & 14,8 & 14,2 & 14,2 & $\mathbf{1 4 , 1}$ & 14,3 & $\mathbf{1 4 , 4}$ \\
\hline Sgo. del Estero & 14,2 & 14,1 & 13,6 & 13,7 & $\mathbf{1 3 , 1}$ & 13,4 & 13,8 & $\mathbf{1 3 , 7}$ \\
\hline La Rioja & 13,0 & 12,5 & $\mathbf{1 1 , 7}$ & 12,2 & 11,8 & 12,0 & 12,6 & $\mathbf{1 2 , 3}$ \\
\hline Ceres & 13,3 & 12,7 & 12,9 & 13,1 & 13,0 & $\mathbf{1 2 , 5}$ & 12,7 & $\mathbf{1 2 , 9}$ \\
\hline Sáenz Peña & 15,9 & $\mathbf{1 5 , 5}$ & 16,4 & 16,5 & 16,0 & 15,6 & 15,6 & $\mathbf{1 5 , 9}$ \\
\hline Posadas & 16,6 & 16,7 & $\mathbf{1 6 , 2}$ & 16,5 & 16,5 & 16,4 & $\mathbf{1 7 , 0}$ & $\mathbf{1 6 , 6}$ \\
\hline La Quiaca & 4,7 & 5,0 & 4,6 & 4,6 & 4,8 & 4,7 & s/d & s/d \\
\hline Rivadavia & 17,8 & 17,3 & 16,8 & 16,9 & 16,8 & 16,5 & s/d & s/d \\
\hline
\end{tabular}

FUENTE: cuadro elaborado a partir de los datos proporcionados por el sitio web NASA-GISS (Goddard Institute for Space Studies)

Los inviernos más cálidos, predominan notoriamente en las décadas de 1931/40 y 1941/50, mientras que los inviernos más fríos se concentran indudablemente en las décadas 1971/50 y 1981/90.

Temperatura media de Primavera. La primavera comprende los meses de Septiembre-OctubreNoviembre, y se distingue por poseer tendencias lineales muy suaves, con excepción de La Rioja y, en menor medida, Posadas, en donde son más marcadas.

De la observación de las tendencias polinómicas se puede concluir que la temperatura media de primavera posee características similares a las ya enunciadas, en cuanto al comportamiento temporal y espacial. Se advierte al mismo tiempo, que a partir de aproximadamente 1995, los registros tienden al aumento en las estaciones Posadas, Ceres y posiblemente Sáenz Peña, mientras que en Corrientes, Salta, Paso de Los libres, Santiago del Estero y La Rioja, muestra suaves descensos. 
TEMPERATURA MEDIA ANUAL DE INVIERNO (en ${ }^{\circ} \mathrm{C}$ ) - PERIODO 1931/2005
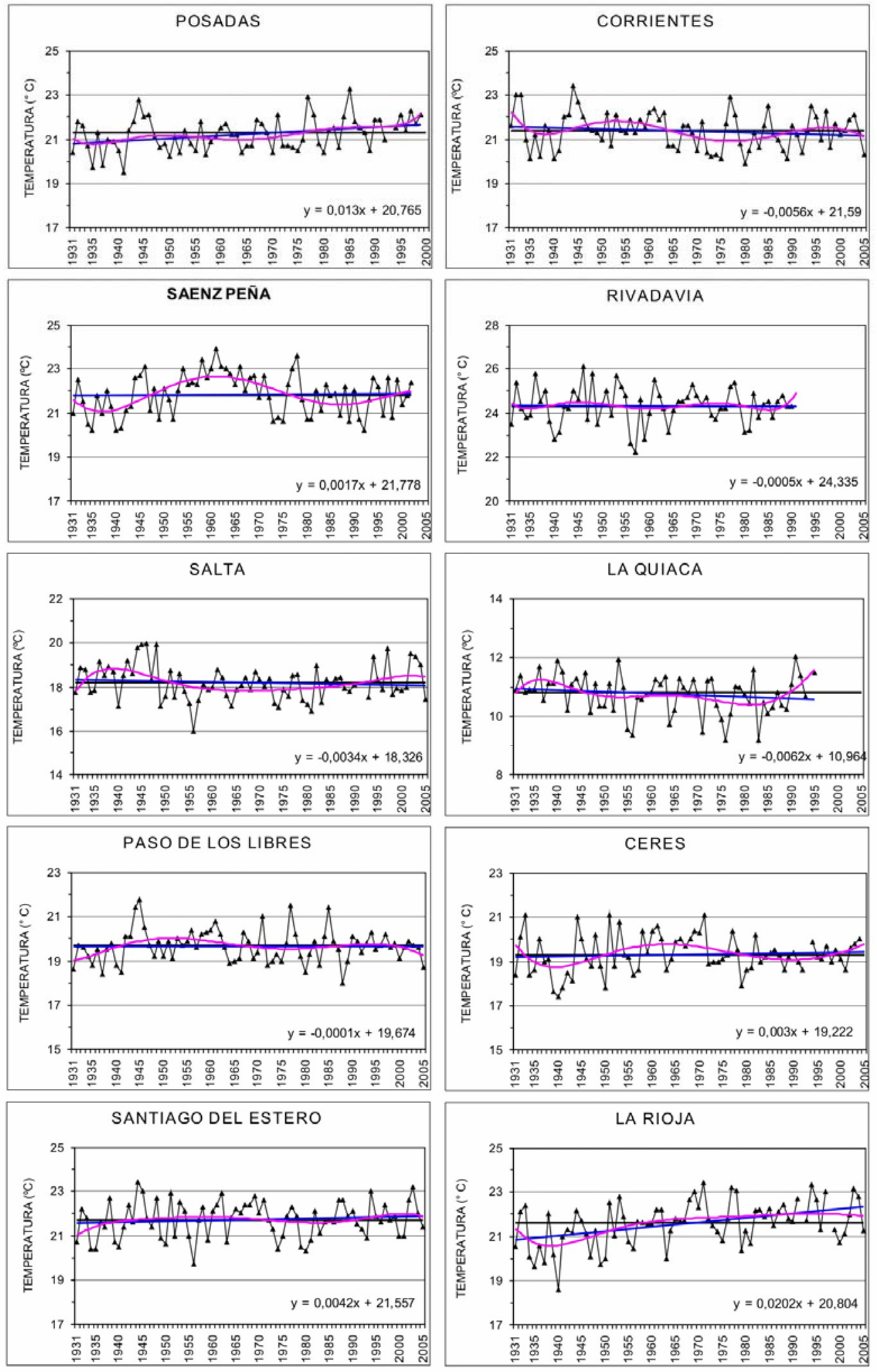

REFERENCIAS

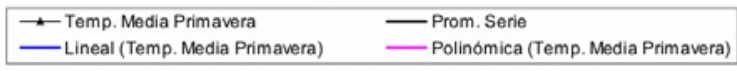

FUENTE: NASA-GISS (Goddard Institute for Space Studies) 


\section{FIGURA 8. Temperatura Media de Primavera - Período 1931/2005}

En cuanto a las diferencias interanuales, se advierte que las mismas son relativamente pequeñas, escasamente superan los dos grados y muestran además, características muy parecidas a las ya mencionadas.

Los valores medios anuales decenales, tal como se advierte en la tabla a continuación (cuyos valores más altos y bajos se han resaltado con color rojo y azul respectivamente), indican que una distribución temporal más dispar de los mismos, de manera similar a los valores de otoño.

TABLA 7. TEMPERATURA MEDIA DECENAL DE PRIMAVERA - PERIODO 1931/1990

\begin{tabular}{|l|c|c|c|c|c|c|c|c|}
\hline \multicolumn{1}{|c|}{ ESTACION } & $\mathbf{1 9 3 1 / 4 0}$ & $\mathbf{1 9 4 1 / 5 0}$ & $\mathbf{1 9 5 1 / 6 0}$ & $\mathbf{1 9 6 1 / 7 0}$ & $\mathbf{1 9 7 1 / 8 0}$ & $\mathbf{1 9 8 1 / 9 0}$ & $\mathbf{1 9 9 1 / 0 0}$ & $\begin{array}{c}\text { PROM. } \\
\text { SERIE }\end{array}$ \\
\hline Salta & 18,6 & $\mathbf{1 8 , 9}$ & $\mathbf{1 7 , 7}$ & $\mathbf{1 8 , 1}$ & 17,8 & 18,0 & $\mathbf{1 8 , 3}$ & $\mathbf{1 8 , 2}$ \\
\hline Corrientes & $\mathbf{2 1 , 3}$ & $\mathbf{2 1 , 8}$ & 21,7 & 21,3 & $\mathbf{2 1 , 0}$ & 21,1 & $\mathbf{2 1 , 5}$ & $\mathbf{2 1 , 4}$ \\
\hline P. de los Libres & $\mathbf{1 9 , 2}$ & $\mathbf{2 0 , 0}$ & 20,0 & 19,6 & 19,6 & 19,6 & 19,7 & $\mathbf{1 9 , 7}$ \\
\hline Sgo. del Estero & $\mathbf{2 1 , 3}$ & 21,9 & 21,6 & $\mathbf{2 2 , 2}$ & 21,4 & 21,8 & 21,8 & $\mathbf{2 1 , 7}$ \\
\hline Ceres & $\mathbf{1 9 , 0}$ & 19,1 & 19,6 & $\mathbf{1 9 , 9}$ & 19,3 & 19,2 & 19,2 & $\mathbf{1 9 , 3}$ \\
\hline Sáenz Peña & $\mathbf{2 1 , 2}$ & 21,7 & 22,3 & $\mathbf{2 2 , 7}$ & 21,8 & 21,6 & 21,5 & $\mathbf{2 1 , 8}$ \\
\hline La Rioja & $\mathbf{2 0 , 6}$ & 20,9 & 21,6 & 21,9 & 21,8 & 21,9 & $\mathbf{2 2 , 1}$ & $\mathbf{2 1 , 5}$ \\
\hline Posadas & $\mathbf{2 0 , 8}$ & 21,2 & 21,0 & 21,1 & 21,2 & 21,6 & $\mathbf{2 1 , 8}$ & $\mathbf{2 1 , 2}$ \\
\hline La Quiaca & 11,1 & 10,8 & 10,6 & 10,9 & 10,4 & 10,5 & $\mathrm{~s} / \mathrm{d}$ & s/d \\
\hline Rivadavia & 24,3 & 24,5 & 23,9 & 24,6 & 24,3 & 24,2 & s/d & s/d \\
\hline
\end{tabular}

FUENTE: cuadro elaborado a partir de los datos proporcionados por el sitio web NASA-GISS (Goddard Institute for Space Studies)

La distribución temporal de los valores nos permite afirmar que las primaveras más cálidas se encuentran durante las décadas de 1940, 1950 y 1960, siempre con la excepción de Posadas y La Rioja, pero al mismo tiempo, en las décadas de 1930, 1940, 1950 y 1970, para las mismas localidades se registraron las marcas más bajas.

A manera de conclusión, se puede destacar que el comportamiento temporal y espacial de la temperatura media estacional posee las siguientes particularidades:

- El verano es la estación más estable del año en cuanto presenta las fluctuaciones o diferencias interanuales más pequeñas, mientras que

- El invierno, por el contrario, es la estación que presenta las fluctuaciones interanuales más marcadas.

- El otoño y la primavera presentan una variabilidad intermedia entre ambos.

Estas características estarían estrechamente vinculadas a los patrones de la circulación atmosférica en el norte argentino dada su condición de encrucijada climática o de transición, con influencia de las zonas de circulación tanto de los alisios como de los oestes, situación que hace que se proyecten esporádicamente sobre el mismo, condiciones meteorológicas habituales de las zonas tropical y templada. El caso particular de Posadas, podría deberse a la localización de la estación, ya que tuvo cambios de emplazamiento en varias oportunidades durante el período de estudio o a cambios ambientales en el entorno, como a posible influencia urbana.

\section{c. La variabilidad de la temperatura}

Las diferencias o fluctuaciones que experimentan de un año para otro los factores climáticos pueden enmascarar cambios graduales o a largo plazo que se producen en el tiempo. El efecto de las "irregularidades" a largo plazo puede eliminarse con diversas técnicas estadísticas; la más simple y utilizada de todas ellas es la "media o promedio móvil", también denominada "media o promedio suavizado" "método que consiste en calcular los valores medios para períodos consecutivos de 5 , 11, 15 ó más años, de acuerdo con la longitud de la serie que se posea. ${ }^{9}$ 
Con este método se suavizan las fluctuaciones a corto plazo si se utilizan períodos de veinte o treinta años, y se ponen de relieve las tendencias a mediano y largo plazo cuando se utilizan series superiores a treinta años.

En los gráficos de la Figura 9, se ha representado las medias móviles de 7 términos de los registros del norte argentino. Saltan a la vista las fluctuaciones de corto período, que abarcan según las estaciones de 10 a 20 años (las fluctuaciones de más largo término y menor amplitud se evidencian en Posadas, Corrientes, Paso de los Libres Ceres, Salta y La Quiaca; mientras que se advierten fluctuaciones de menor escala temporal, pero de mayor amplitud y marcada periodicidad (ciclos?) en Santiago del Estero y La Rioja, por ejemplo)

Esta técnica estadística refleja, nuevamente y con gran claridad, que los años 1930 y 1940 fueron los más cálidos en la mayoría de estas estaciones. También permite advertir la presencia de modelos o patrones de comportamiento, que indican la presencia de dos períodos cálidos separados por uno más frío en Posadas, Corrientes, Rivadavia, Salta, La Quiaca, Paso de los Libres (a pesar de algunas diferencias menores, tales como que, en Corrientes es más marcado el calentamiento de la primer mitad de la serie, mientras que en Posadas lo es en la segunda mitad de la misma, en Paso de los Libres las fluctuaciones son mucho más suaves, entre otras); la presencia de un período cálido muy marcado en el medio de la serie para los casos de Sáenz Peña y Ceres y la existencia de tres ciclos cálidos bien marcados en Santiago del Estero y La Rioja. 
TEMPERATURA MEDIA ANUAL (en ${ }^{\circ} \mathrm{C}$ ) - PROMEDIOS SUAVIZADOS (de 7 y 15 términos) - PERIODO 1931/2005
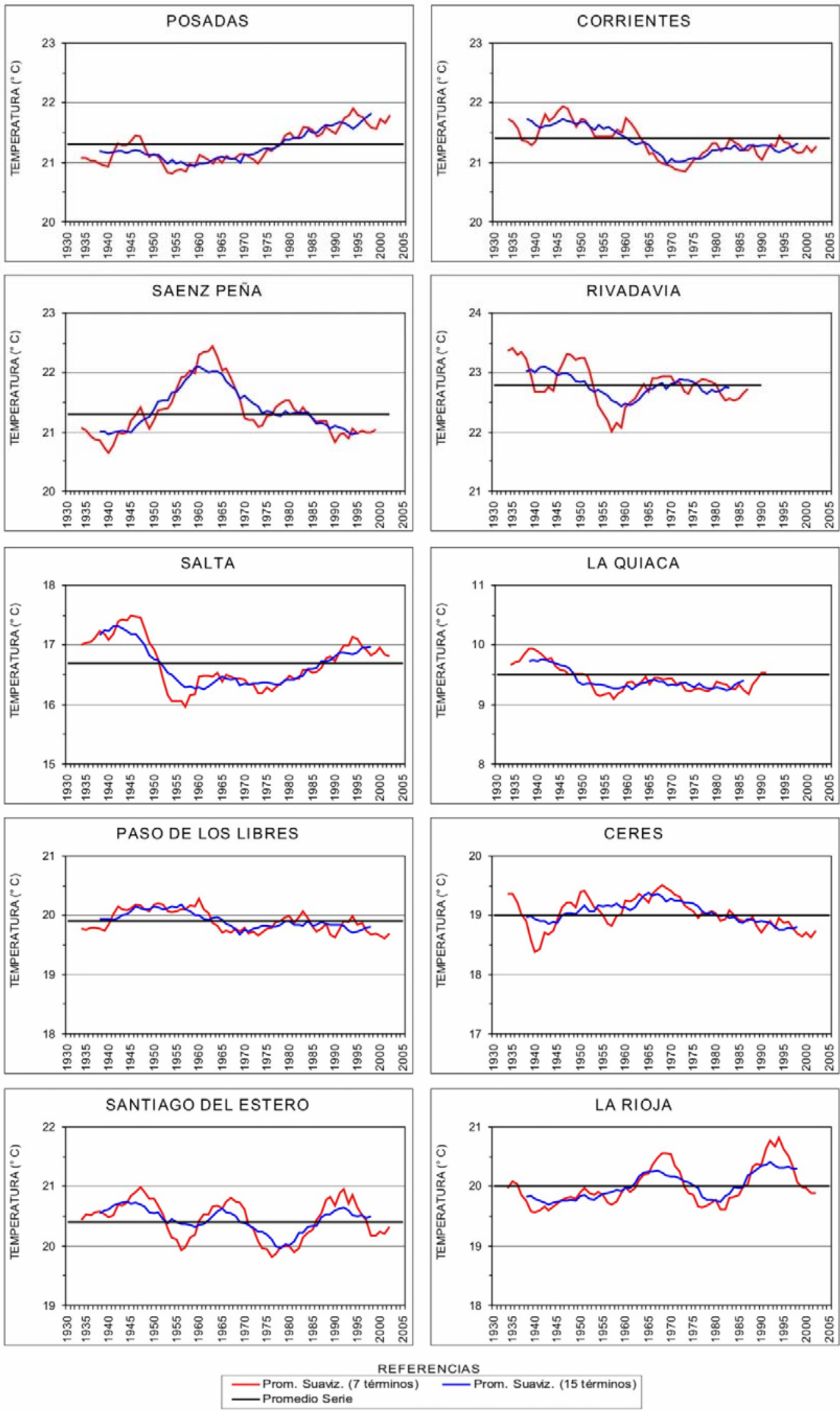

FUENTE: NASA-GISS (Goddard Institute for Space Studies) 
FIGURA 9. Temperatura Media Anual - Promedios Suavizados de 7 y 15 términos (en $\left.{ }^{\circ} \mathrm{C}\right)$ Período 1931/2005

Las tendencias a más largo plazo (multidecenales y seculares), se hacen más evidentes cuando se utilizan las medias suavizadas de 15 términos, que en reglas generales reflejan condiciones idénticas a las anteriores, en lo que se refiere a los modelos o patrones de comportamiento y también en la alternancia de períodos más cálidos que alternan con otros más fríos, pero siempre en torno al valor medio. ${ }^{10}$

Otra manera de medir las fluctuaciones y variaciones temporales (por ejemplo de un mes, estación o año determinados) y espaciales es mediante las "anomalías o desviaciones desde la normal", que nos indican la "variabilidad climática" de un lugar, región, hemisferio o el planeta en su totalidad.

La variabilidad se puede medir de diferentes maneras; una de ellas es mediante las diferencias existentes entre un mes, estación o año en particular con respecto del "promedio de la serie" o, de un período en particular, tomado como "base de referencia". Dichas diferencias, que pueden ser negativas y/o positivas, son consideradas anomalías o desviaciones desde la normal, entendiéndose por normal el valor medio o promedio de la serie, y fueron graficadas en la Figura 10. 

$G_{0} \begin{aligned} & \text { Revista Geográfica Digital. IGUNNE. Facultad de Humanidades. UNNE. Año 3- No } 6 \\ & \text { Julio - Diciembre 2006. ISSN 1668-5180. Resistencia, Chaco }\end{aligned}$

DESVIACIONES DE LA TEMPERTURA MEDIA ANUAL DESDE LA NORMAL (en ${ }^{\circ}$ C) - PERIODO 1931/2005
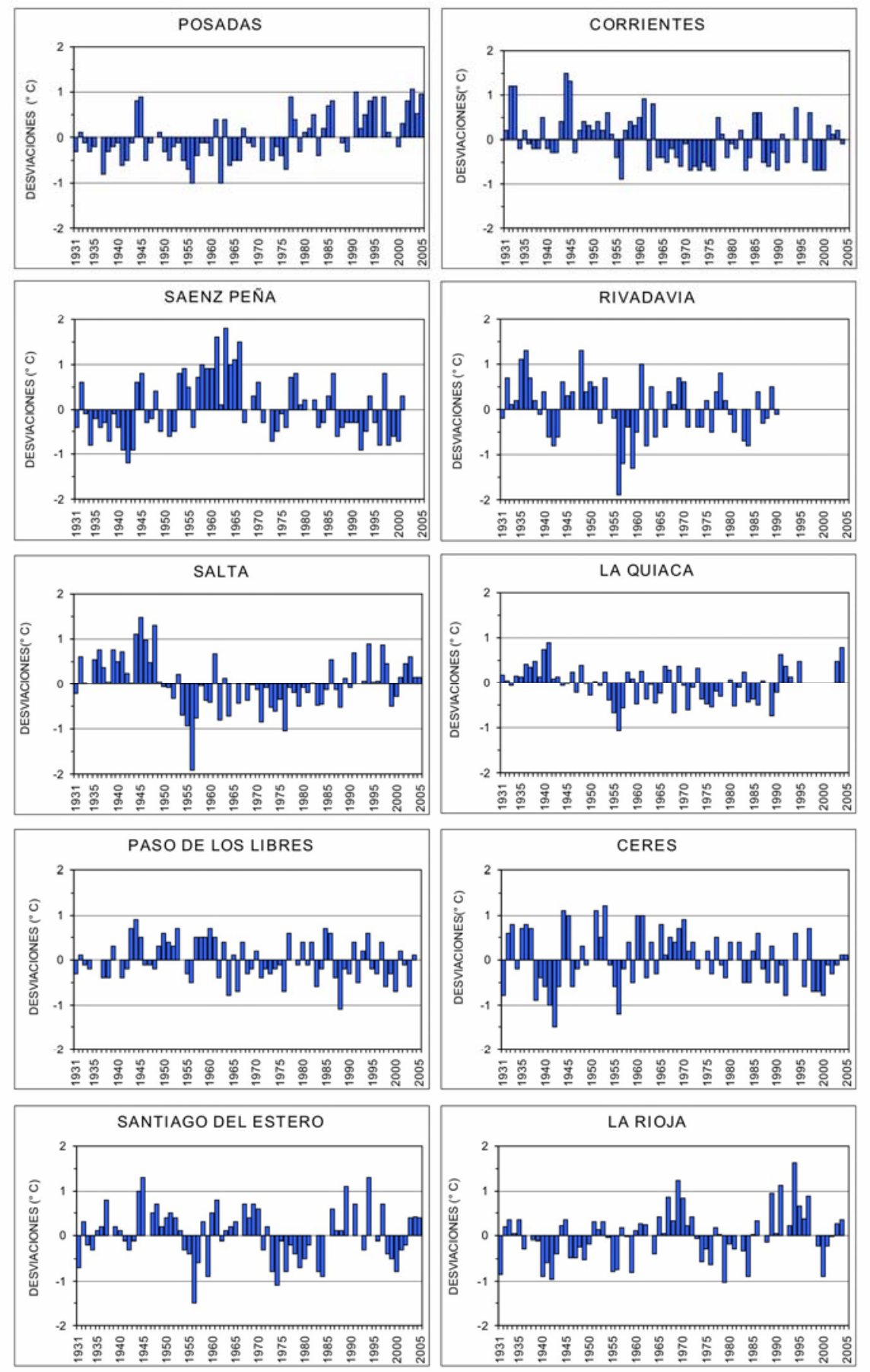

REFERENCIAS

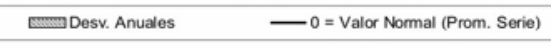

FUENTE: NASA-GISS (Goddard Institute for Space Studies) 


\section{FIGURA 10. Temperatura Media Anual - Desviaciones desde la Normal (en ${ }^{\circ} \mathrm{C}$ ) - Período $1931 / 2005$}

Las anomalías de la temperatura media anual muestran, obviamente, características similares a las indicadas para el comportamiento temporal y espacial de la temperatura media anual y estacional. Lo más destacable de dicho comportamiento es:

- La variabilidad interanual de la temperatura a lo largo del período de estudio, no muestra aumentos significativos como se afirma en numerosos trabajos de investigación, que utilizan este dato como una señal del probable cambio climático.

- Por el contrario, es evidente que en las estaciones Corrientes, Sáenz Peña, Ceres, La Quiaca, y Santiago del Estero, ha disminuido durante los últimos años, siendo mucho más marcada durante la primera mitad de la serie.

- En aquellas estaciones en que la variabilidad ha aumento durante los últimos años, tales como Posadas, el aumento detectado es poco marcado.

- En La Rioja los años 1990 fueron los de mayor variabilidad, pero a partir del 2000 desciende abruptamente.

- En Paso de los Libres se mantiene sin mayores variaciones, si bien durante los primeros años de la serie la variabilidad fue mucho menor.

- Por último, en términos generales, la variabilidad de la temperatura es menor en las estaciones situadas al oriente del área de estudio, se acentúa en las del centro y alcanza los mayores valores en las localidades del occidente, con la excepción de La Quiaca, en la cual intervienen otros factores, tales como la altura y la latitud, para mantener una relativa estabilidad climática a lo largo del período de estudio.

En cuanto a las desviaciones desde la normal de la temperatura media decenal, valen las mismas consideraciones que para las desviaciones interanuales.

TABLA 8. DESVIACIONES DE LA TEMPERATURA MEDIA ANUAL DECENAL DESDE LA NORMAL- PERIODO 1931/1990

\begin{tabular}{|c|c|c|c|c|c|c|c|c|}
\hline ESTACION & $1931 / 40$ & $1941 / 50$ & $1951 / 60$ & $1961 / 70$ & $1971 / 80$ & $1981 / 90$ & $1991 / 00$ & $\begin{array}{l}\text { PROM. } \\
\text { SERIE }\end{array}$ \\
\hline \multirow{2}{*}{ Salta } & 17,0 & 17,3 & 16,2 & 16,5 & 16,3 & 16,6 & 16,9 & 16,7 \\
\hline & 0.3 & 0.6 & -0.5 & -0.2 & -0.4 & -0.1 & 0.2 & \\
\hline \multirow{2}{*}{ Corrientes } & 21,6 & 21,7 & 21,5 & 21,2 & 20,9 & 21,2 & 21,2 & 21,3 \\
\hline & 0.3 & 0.4 & 0.2 & -0.1 & -0.4 & -0.1 & -0.1 & \\
\hline \multirow{2}{*}{ Sgo, del Estero } & 20,4 & 20,8 & 20,2 & 20,8 & 19,9 & 20,4 & 20,4 & 20,4 \\
\hline & 0.0 & 0.4 & -0.2 & 0.4 & -0.5 & 0.0 & 0.0 & \\
\hline \multirow{2}{*}{ P. de los Libres } & 19,7 & 20,1 & 20,2 & 19,8 & 19,8 & 19,8 & 19,8 & 19,9 \\
\hline & -0.2 & 0.2 & 0.3 & -0.1 & -0.1 & -0.1 & -0.1 & \\
\hline \multirow{2}{*}{ Sáenz Peña } & 21,0 & 21,1 & 21,7 & 22,1 & 21,3 & 21,2 & 20,9 & 21,3 \\
\hline & -0.3 & -0.2 & 0.4 & 0.8 & 0.0 & -0.1 & -0.4 & \\
\hline \multirow{2}{*}{ Ceres } & 19,1 & 18,8 & 19,2 & 19,4 & 19,1 & 18,9 & 18,7 & 19,0 \\
\hline & 0.1 & -0.2 & 0.2 & 0.4 & 0.1 & -0.1 & -0.3 & \\
\hline \multirow{2}{*}{ La Rioja } & 19,9 & 19,7 & 19,9 & 20,4 & 19,8 & 20,0 & 20,4 & 20,0 \\
\hline & -0.1 & -0.3 & -0.1 & 0.4 & -0.2 & 0.0 & 0.4 & \\
\hline \multirow{2}{*}{ Posadas } & 21,1 & 21,3 & 20,9 & 21,1 & 21,2 & 21,4 & 21,7 & 21,2 \\
\hline & -0.1 & 0.1 & -0.3 & -0.1 & 0.0 & 0.2 & 0.5 & \\
\hline
\end{tabular}

FUENTE: cuadro elaborado a partir de los datos del sitio web NASA-GISS (Goddard Institute for Space Studies)

En este caso hay que destacar que la variabilidad nuevamente nos está determinando, a través de sus valores, las décadas en las cuales la misma fue más marcada y las décadas más cálidas y frías del período.

\section{CONCLUSIONES}


1. Los registros indican que durante los últimos 150 años, la temperatura se ha incrementado en la mayor parte del planeta, calentamiento que es del orden 0.3 a $0.6^{\circ} \mathrm{C}$, según diferentes estimaciones y ajustes, pero este incremento no ha sido geográficamente uniforme ni continuo en el tiempo.

2. En el Hemisferio Sur las temperaturas también se han incrementado desde finales del siglo XIX, pero en menor medida, el aumento es de $0.2^{\circ}$ a $0.3^{\circ} \mathrm{C}$.

3. Las series anuales de ambos Hemisferios muestran algunas similitudes generales, tales como: tendencias al calentamiento antes de 1880, al que continúa un descenso hasta aproximadamente 1915, seguido de un nuevo ascenso hasta principios de 1940. A partir de esta fecha, se registra otro descenso entre 1950 y 1980, para ascender marcadamente hasta nuestros días, registrándose los mayores valores con posterioridad a 1980.

4. Las series anuales del Hemisferios Sur también muestran algunas diferencias marcadas, con el Hemisferio Norte, tales como: el calentamiento observado con anterioridad a 1880 es menor y también son menores los descensos y aumentos que se observan en los distintos períodos mencionados. Otra diferencia a destacar que los períodos no siempre son exactamente coincidentes.

5. Varias de las características determinadas a nivel global y hemisférico, no se verifican en las estaciones del norte argentino, salvo los casos de Posadas y la Rioja, ya que:

- los registros indican que las décadas más calientes en los últimos 75 años fueron las de 1930/1940/1950 y 1960, por otra parte,

- si bien las últimas décadas han registrado un aumento de la temperatura hasta el año 2000, en general, las actuales temperaturas permanecen por debajo de las reportadas hace 70 años, además,

- la temperatura no presenta un aumento continuo y sostenido desde 1931 hasta nuestros días, sino que por el contrario, se advierte una alternancia de décadas más cálidas con otras más frías (siempre con la excepción de Posadas).

5. Las diferencias detectadas podrían originarse en los siguientes aspectos:

- la longitud de las series analizadas y el período que se toma como base de referencia para la temperatura media a partir de la cual se calculan las desviaciones. En las investigaciones hemisféricas y planetarias el período de registros cubre los años 1856/2005, pero las anomalías están referidas al promedio del período 1961/1990, que según indican fue más cálido que los anteriores en la mayor parte del globo, con lo cual las anomalías/desviaciones negativas, anteriores a 1960 se acentúan,

- actualmente, la mayor disponibilidad de datos permitió comprobar que las fluctuaciones/variaciones no están distribuidas al azar en el espacio y en el tiempo sino que, a menudo, aparecen como si estuvieran organizadas dentro de una estructura espacial relativamente coherente, mientras que su amplitud, fases y posición geográfica cambian/varían a través del tiempo. Aunque la naturaleza y forma de esas estructuras o patrones varía, de acuerdo con la metodología estadística y las series empleadas en el análisis, se advierten características regionales consistentes que identifican patrones o modelos, que reflejan que el clima posee fluctuaciones y/ variaciones en el tiempo y en el espacio, tal como sucede en el norte de nuestro país,

- finalmente, no podemos dejar de mencionar la fuerte influencia moderadora de los océanos del sur, a que está sujeto el extremo sur de América del Sur y en especial nuestro país, que se hace evidente en la menor variación que poseen los datos de observación en estas regiones.

\section{NOTAS Y CITAS BIBLIOGRAFICAS}

1. De esta clasificación elaborada por la Organización Meteorológica Mundial, que se transcribió de Cuadrat, José M. y Pita, María F. (1997). Climatología. Madrid, Cátedra. Anexo I al Capítulo 10, pp. 481/482, surgen los siguientes interrogantes:

a. ¿cambio climático es toda/cualquier diferencia producida en los valores, sin tener en cuenta su naturaleza, causa o magnitud espacial y temporal, frecuencia, etc?

b. ¿Si una discontinuidad es un cambio, cuál es la extensión del período de observación: un año, diez, treinta, cien, etc.?

c. ¿Si la fluctuación es una inconstancia climática, fluctuación es lo mismo que cambio climático?

d. ¿La oscilación climática también es una fluctuación? 
e. Si la periodicidad climática es un ritmo, el ritmo climático es una oscilación o vacilación, y la vacilación climática es una fluctuación, significa que no hay diferencias entre estos tres tipos de inconstancia climática, es todo lo mismo?

f. Nuevamente se habla de un cambio climático sin aclarar la magnitud de los valores ni la duración del período de observación, por otra parte, a qué se hace referencia con: "caracterizado por un solo máximo y un solo mínimo en los puntos finales de la serie"?

g. ¿De la misma manera, si una vacilación climática es al mismo tiempo una fluctuación climática, y una variación climática también es una fluctuación, qué se debe entender, en sentido estricto, por fluctuación climática?

h. ¿Reiteradamente, una variación climática también es una fluctuación?

2. Cf. Intergovernmental Panel on Climate Change. En: Third Assessment Report, Climatic Change 2001. The Scientific Basis. Cap. 2, Figuras 2.9.a a 2.9.d. Annual Temperatura Trends. 1901/2000; 1910/1945; 1946/1975 y 1976/2000, p. 116.

3. El sitio web de la NASA-GISS Surface Temperature Analysis (GISTEMP) cuenta con las series históricas de la temperatura de superficie, con resolución mensual a partir de 1880, cuando se estableció una distribución global razonable de las estaciones meteorológicas. Los datos para el análisis provienen originalmente de los servicios meteorológicos nacionales de alrededor del mundo, que son posteriormente revisados, corregidos y actualizados mensualmente. Este sitio proporciona datos de unas 3000 estaciones, registros que han sido corregidos y normalizados para salvar errores tales como saltos o discontinuidades, debidos a cambios en los instrumentos y a la relocalización de las estaciones.

4. La recta de tendencia lineal consiste en una línea recta que se ajusta perfectamente y que se utiliza, generalmente, con conjuntos de datos lineales simples, es decir, datos que se agrupan alrededor de una recta. Ahora, una línea de tendencia lineal normalmente muestra que algo aumenta o disminuye a un ritmo constante, hecho que no se observa en las representaciones, aún en aquellas estaciones con las tendencias más marcadas, y eso es debido a las fluctuaciones interanuales y decenales que presenta la temperatura a lo largo del período de estudio.

5. El aumento de temperatura registrado en la estación Posadas podría deberse a los continuos cambios de emplazamiento que sufriera la misma y a los cambios ambientales a que estuvo sujeto el área de emplazamiento.

6. La curva de tendencia polinómica es una línea curva que se utiliza cuando los datos fluctúan alrededor de un valor medio, tal como lo hace la temperatura. El orden del polinomio se puede determinar mediante el número de fluctuaciones en los datos o en función del número de máximos y mínimos que aparecen en la curva, o bien, de la longitud de la serie. Por ejemplo, una línea de tendencia polinómica de Orden 2 suele tener sólo un máximo o un mínimo, una de Orden 3 normalmente tiene uno o dos máximos o mínimos, la de Orden 4 tiene más de tres, y así sucesivamente. En este caso hemos optado por la de 6 términos.

7. A nivel planetario y hemisférico, los registros indican como el año más cálido de la serie 1880/2005, el de 1998. Cf. Intergovernmental Panel on Climate Change. En: Third Assessment Report, Climatic Change 2001. The Scientific Basis. Cap. 2, Fig. 2.7, p. 114.

8. El año 1956 se destaca como el más frío en las localidades citadas, especialmente en las localizadas en el occidente del área de estudio (Rivadavia, Salta, La Quiaca y Santiago del Estero) en las cuales el descenso fue notoriamente marcado $\left(1^{\circ} \mathrm{C}\right.$ en Salta, $1.7^{\circ} \mathrm{C}$ en Rivadavia, $1.1^{\circ} \mathrm{C}$ en Santiago del Estero), de acuerdo con el enfriamiento que se produjo durante la década 1950 en el Noroeste de nuestro país, condición que también prevaleció en gran parte del norte y centro del territorio nacional. Cf. Minetti, J. L. y Sal Paz, J. C. 1981. El enfriamiento regional del NorOeste Argentino a partir de la década de 1950. Tucumán (Argentina), EEAOC.

9. Las medias móviles o suavizadas pueden calcularse sobre una base de 3, 5, 7, 9, 11 años o más, lo cual depende de la longitud de la serie considerada y de las tendencias a determinar (decenales, multidecenales, seculares, etc.). En esta oportunidad, y dada la duración del período en estudio, hemos calculado los promedios móviles sobre la base de siete (7) y once (11) años, a efectos de destacar las tendencias de corto y medio plazo. Para su cálculo, se procede de la siguiente manera:

año $1+$ año $2+$ año $3+$ año $4+$ año $5+$ año $6+$ año $7=$ el promedio se asigna al año 4 
año $2+$ año $3+$ año $4+$ año $5+$ año $6+$ año $7+$ año $8=$ el promedio se asigna al año 5

7

10. Por razones de espacio no hemos incluido los gráficos correspondientes a los promedios suavizados estacionales, pero valen las mismas apreciaciones realizadas en el análisis de la temperatura media anual y estacional.

\section{BIBLIOGRAFIA}

- BARRY, R. y CHORLEY, R. (1985). Atmósfera, tiempo y clima. Barcelona, Omega.

- BIROT, Pierre. (1963). Tratado de Geografía Física. Barcelona, Vicens Vives.

- BRUNIARD, E. D. (1992). El ámbito subtropical en la República Argentina (Climatología dinámica y límites climáticos. En: Estudios Geográficos. Madrid, № 208.

- BRUNIARD, E., PEREZ, M. E., GONZALEZ, C. D. Y BIANCONI, A. (2001). El Clima de la Región Algodonera Argentina. Informe Elaborado para el Proyecto Integral Algodonero. Resistencia, Convenio Gobierno de la Provincia del Chaco - UNNE.

- CAPEL MOLINA, José Jaime. (1999). "EL Niño" y el sistema climático terrestre. Barcelona, Ariel.

- CUADRAT, José María y PITA, María Fernanda. (1997). Climatología. Madrid, Cátedra.

- DIAZ, Henry F. y BRADLEY, Raymond S. (1995). Documentando las Variaciones Climáticas Naturales: ¿Cuán Diferente es el Clima del Siglo Veinte con el de Siglos Anteriores? En: Natural Climate Variability on Decade-to-Century Time Scales. Washington, National Academy Press.

- FERNANDEZ GARCIA, Felipe. (1996). Manual de Climatología Aplicada. Clima, Medio Ambiente y Planificación. Madrid, Síntesis.

- FLOHN, Hermann. (1974). Climatic Variation and Modification of Climate. Facts and Problems. En: Applied Sciences and Development. Tübingen, Institute for Scientific Cooperation, Vol. 3.

- HOFFMANN, José A. (1988). Las variaciones climáticas ocurridas en la Argentina desde fines del siglo pasado hasta el presente. En: El deterioro del ambiente en la Argentina (suelo, agua, vegetación, fauna) Buenos Aires, Fundación para la educación la ciencia y la cultura ( $\mathrm{FECIC}$ ).

- HUFTY, A. (1984). Introducción a la Climatología. Barcelona, Ariel.

- JONES, P. D. (1994). Hemispheric Surface Air Temperature Variations: a reanalysis anda $n$ update to 1993. En: Journal of Climate, vol. 7.

- KARL, Thomas R. (1995). Observaciones Atmosféricas: Un Ensayo. En: Natural Climate Variability on Decade-to-Century Time Scales. Washington, National Academy Press.

- KARL, T. R., KNIGHT, R. W. and CHRISTY, J. R. (1994). Global and Hemispheric Temperature Trends: uncertainties related to inadequate spatial sampling. En: Jounal of Climate, vol. 7.

- MARIN, Mercedes. (1990). Normalidad y Anomalía en la Investigación Climatológica Actual. En: Revista de Geografía. Barcelona, Dpto. de Geografía, Vol. XXIV.

- MINETTI, J. L. y W. M. Vargas. (1983). El Enfriamiento de la Década de 1950 en la R.A. En: Meteorológica. Buenos Aires, Centro Argentino de Meteorólogos, Vol.14.

- MINETTI, J., VARGAS, W., HERNANDEZ, C. y LOPEZ, E. (2002). La circulación regional estacional en Sud América. En: Breves Contribuciones del I.E.G. Tucumán, Universidad Nacional de Tucumán, Facultad de Filosofía y Letras.

- NATIONAL ACADEMY PRESS (1995). Natural Climate Variability on Decade-to-Century Time Scales. Washington, D.C., Climate Research Committee Board on Atmospheric Sciences and Climate Commission on Geosciences, Environment, and Resources National Research Council.

- PANEL INTERGUBERNAMENTAL SOBRE CAMBIO CIMATICO (2001). Cambio Climático 2001. La base científica. Tercer Informe de Evaluación. OMM-PNUMA.

- RICCIARDI, H. J. (1995). Cambio Global. Causas, ciencia, tecnología e implicaciones humanas. Buenos Aires, Academia Nacional de Geografía, Publicación Especial № 10.

- STRAHLER, Arthur y STRAHLER, Alan. (1990). Geografía Física. $3^{\circ}$ ed. Barcelona, Omega. 